\title{
Trapped-mode-induced Fano resonance and acoustical transparency in a one-dimensional solid-fluid phononic crystal
}

\author{
Ilyasse Quotane, ${ }^{1}$ El Houssaine El Boudouti, ${ }^{1,}{ }^{*}$ and Bahram Djafari-Rouhani ${ }^{2}$ \\ ${ }^{1}$ Laboratoire de Physique de la Matière et du Rayonnement (LPMR), Département de Physique, Faculté des Sciences, \\ Université Mohammed I, Oujda, Morocco \\ ${ }^{2}$ Institut d'Electronique, de Microélectronique et de Nanotechnologie (IEMN), UMR CNRS 8520, UFR de Physique, \\ Université des Sciences et Technologies de Lille, 59655 Villeneuve d'Ascq, France
}

(Received 15 October 2017; revised manuscript received 31 December 2017; published 16 January 2018)

\begin{abstract}
We investigate theoretically and numerically the possibility of existence of Fano and acoustic-induced transparency (AIT) resonances in a simple though realistic one-dimensional acoustic structure made of solid-fluid layers inserted between two fluids. These resonances are obtained by combining appropriately the zeros of transmission (antiresonance) induced by the solid layers and the local resonances induced by the solid or combined solid-fluid layers with surface free boundary conditions. In particular, we show the possibility of trapped modes, also called bound states in continuum, which have recently found a high renewal interest. These modes appear as resonances with zero width in the transmission spectra as well as in the density of states (DOS). We consider three different structures: (i) a single solid layer inserted between two fluids. This simple structure shows the possibility of existence of trapped modes, which are discrete modes of the solid layer that lie in the continuum modes of the surrounding fluids. We give explicit analytical expressions of the dispersion relation of these eigenmodes of the solid layer which are found independent of the nature of the surrounding fluids. By slightly detuning the angle of incidence from that associated to the trapped mode, we get a well-defined Fano resonance characterized by an asymmetric Fano profile in the transmission spectra. (ii) The second structure consists of a solid-fluid-solid triple layer embedded between two fluids. This structure is found more appropriate to show both Fano and acoustic-induced transparency resonances. We provide detailed analytical expressions for the transmission and reflection coefficients that enable us to deduce a closed-form expression of the dispersion relation giving the trapped modes. Two situations can be distinguished in the triple-layer system: in the case of a symmetric structure (i.e., the same solid layers) we show, by detuning the incidence angle $\theta$, the possibility of existence of Fano resonances that can be fitted following a Fano-type expression. The variation of the Fano parameter that describes the asymmetry of such resonances as well as their width versus $\theta$ is studied in detail. In the case of an asymmetric structure (i.e., different solid layers), we show the existence of an incidence angle that enables to squeeze a resonance between two transmission zeros induced by the two solid layers. This resonance behaves like an AIT resonance, its position and width depend on the nature of the fluid and solid layers as well as on the difference between the thicknesses of the solid layers. (iii) In the case of a periodic structure (phononic crystal), we show that trapped modes and Fano resonances give rise, respectively, to dispersionless flat bands with zero group velocity and nearly flat bands with negative or positive group velocities. The analytical results presented here are obtained by means of the Green's function method which enables to deduce in closed form: dispersion curves, transmission and reflection coefficients, DOS, as well as the displacement fields. The proposed solid-fluid layered structures should have important applications for designing acoustic mirrors and acoustic filters as well as supersonic and subsonic materials.
\end{abstract}

DOI: 10.1103/PhysRevB.97.024304

\section{INTRODUCTION}

In several physical problems, the interaction of an incident wave with the scattering centers in a composite material gives rise to resonances as a consequence of constructive interference of the waves. The well-known one is the BreitWigner [1] resonance following a Lorentzian shape. However, some systems may show a resonance followed by an antiresonance (as a consequence of destructive interference of the waves) giving rise to an asymmetric line shape. This kind

*Corresponding author: elboudouti@yahoo.fr of resonance is known as Fano resonance [2,3]. When the resonance falls between two antiresonances, the latter is called electromagnetic-induced transparency (EIT) resonance [4,5]. Fano and EIT resonances are originally described as quantum phenomena. Indeed, Fano resonance is defined as the result of constructive and destructive interferences of a discrete state with a continuum background [2,3], while EIT resonance is the phenomenon where a sharp transparent window (enhanced transmission) associated with steep dispersion is introduced into an opaque medium [4,5]. In addition to Fano and EIT resonances, trapped modes also called bound in continuum (BIC) states have been the subject of several studies [615]. These modes appear as resonances with zero width in 
the transmission spectra as well as in the density of states (DOS). Therefore, they remain confined in some parts of the system even though they coexist with a continuous spectral range of radiating waves that can transport energy away. This is a counterintuitive idea which was originally proposed several decades before [16], but found a high amount of interest in the recent literature [17] because it became possible to propose different realistic or technologically interesting structures displaying such a phenomenon. Indeed, because of their high- $Q$ factor, BIC modes can be used for narrow-band filtering, chemical or biosensing, and low-threshold lasing [17]. These modes without leakage have been found as discrete modes in the band structure of a periodic system, or as a consequence of the transformation of Fano and EIT resonances into hidden resonances with practically infinite lifetimes when their linewidths are reduced to zero [6-15,17].

Although all these resonances were first proposed in quantum mechanics, it was demonstrated that such phenomena can be extended to classical systems such as plasmonic materials and planar metamaterials [18-24], photonic crystal waveguides coupled to cavities [25-29], coupled microresonators [30-35], photonic crystal slabs [14,15], and photonic circuits $[36,37]$. However, little work has been devoted to acoustic counterpart systems [38-49]. A first paper [38] dealing with a theoretical demonstration of trapped, Fano, and EIT resonances in acoustics has been published by some of us in a simple one-dimensional (1D) structure made by two side-detuned resonators grafted at two sites on a tube. These results have been confirmed experimentally in some recent papers showing the possibility to support Fano [41], EIT [39-41], and EIA [42] (electromagnetic-induced absorption) phenomena. Different Fano resonances have been treated also in two-dimensional (2D) locally resonant sonic crystals [46], surface hypersonic crystals [47], pillared acoustic metasurface [48], and acoustical duct cavity [43] as a consequence of the existence of trapped modes [50]. The acoustic analog of EIT resonance has been shown in $2 \mathrm{D}$ phononic crystals made of a periodic array of poly(methyl methacrylate) (PMMA) square rods immersed in water [44] and coupled-pipe resonators [45].

In this paper, we propose a realistic though simple platform constituted by alternating solid-fluid layers to show the possibility of such phenomena. Although these structures have been the subject of many works for their acoustic applications, the existence of Fano, acoustic-induced transparency (AIT), and trapped modes have not been reported before. We demonstrate theoretically that a simple acoustic structure made of a single solid layer or a solid-fluid-solid triple layer immersed in a fluid may support trapped, Fano, and AIT resonances. These resonances are shown through an analysis of the transmission coefficient (amplitude and phase) and DOS obtained using the Green's function method [51]. First, we show the possibility of existence of trapped modes as a consequence of the combination of the transmission zeros induced by the solid layers and discrete modes induced either by a single solid layer or a triple solid-fluid-solid layer with free surfaces for a given incidence angle $\theta_{0}$. Then, a small shift of the incidence angle from $\theta_{0}$ enables to separate the resonance from the transmission zero (antiresonance) giving rise to a Fano resonance in the case of a symmetric structure. Also, breaking properly the symmetry of the structure by detuning for example the thicknesses of the two solid layers enables to squeeze a resonance between two transmission zeros induced by the two different solid layers, giving rise to an AIT resonance. The dependence of these resonances on the nature of the solid and fluid layers is discussed. Finally, we show that in a periodic multilayer solid-fluid structure (i.e., 1D phononic crystal), the trapped modes give rise to a totally flat band with zero group velocity, whereas when they are transformed into Fano resonances, the latter develop into nearly flat bands with nearly zero positive or negative group velocities.

It is worth mentioning that the propagation of acoustic waves in solid-fluid layered media have been designed to demonstrate different phenomena such as Bragg, non-Bragg, and omnidirectional band gaps [52], Bloch oscillations [53], super-wide-angle propagation phenomena about the second critical angle [54], tunable acoustic filter [55,56], one-way acoustic transmission [57,58], and sensors [59].

The paper is organized as follows: In Sec. II, we give a brief review of the method of calculation used in this work which is based on the Green's function method [51]. In Sec. III, we give an analytical demonstration of the so-called trapped mode and we show numerically the possibility of existence of asymmetric Fano resonances in a single solid layer sandwiched between two fluids. Section IV is devoted to a triple solid-fluid-solid layer. Here also, we give analytical expressions of the trapped modes as well as the conditions behind the existence of the so-called Fano and AIT resonances. In Sec. V, we study the propagation of acoustic waves in solid-fluid periodic structures (1D phononic crystal) where the trapped modes and Fano resonances give rise, respectively, to flat bands with zero group velocity and nearly flat bands with positive or negative group velocities. The conclusions are presented in Sec. VI.

\section{THEORETICAL MODEL}

The method of calculation used in this study is based on the interface response theory [51] of continuous media which allows us to calculate the Green's function of any composite system. Let us notice that the Green's function method has the ability of providing in a compact form several dynamical properties of the system such as dispersion curves, transmission/reflection coefficients, densities of states (DOS), displacement fields. In what follows, we very briefly present the basic concepts and the fundamental equations of this theory $[51,60]$, then give the useful relations for the application of the method to the study of the solid-fluid structure. Let us consider a composite material contained in its space of definition $D$. This system contains different subsystems $i$ connected together by their interface domains $M_{i}$. The whole interface space of the system is labeled $M=\cup M_{i}$. The elements of the Green's function $g(D D)$ of any composite material can be obtained from $[51,60]$

$$
\begin{aligned}
g(D D)= & G(D D)-G(D M) G^{-1}(M M) G(M D) \\
& +G(D M) G^{-1}(M M) g(M M) G^{-1}(M M) G(M D),
\end{aligned}
$$

where $G(D D)$ is the Green's function of a homogeneous continuous medium and $g(M M)$ is the Green's function of the composite system in the space $M$ of the interfaces. $D$ is the 
whole space of the system and $M$ is the space of interfaces. As we are interested in elastic waves in solid and fluid media, the corresponding Green's function $G(D D)$ can be derived from the equation of motion of the displacement field as explained in Refs. $[51,60]$. The inverse of the Green's function in the space of interfaces $M[g(M, M)]^{-1}$ is obtained as a superposition of the different $g_{i}^{-1}\left(M_{i}, M_{i}\right)$ for each constituent $i$ of the composite system $[51,60]$. The knowledge of $g(M M)$ allows us to obtain the eigenmodes of a composite system through the relation $[51,60]$

$$
\operatorname{det}\left[g^{-1}(M M)\right]=0,
$$

$U(D)$ being an eigenvector of the reference system [60]. Equation (2) leads to the eigenvectors $u(D)$ of the composite material as

$$
\begin{aligned}
u(D)= & U(D)-U(M) G^{-1}(M M) G(M D) \\
& +U(M) G^{-1}(M M) g(M M) G^{-1}(M M) G(M D) .
\end{aligned}
$$

ln Eq. (3), $U(D), U(M)$, and $u(D)$ are row vectors. If $U(D)$ is a bulk wave launched in one homogeneous piece of the composite material, then Eq. (3) enables the calculation of all the waves reflected and transmitted by the interfaces, as well as the reflection and transmission coefficients of the composite system [60].

In this paper, we are interested in the propagation of acoustic waves polarized in the sagittal plane defined by the normal to the interfaces ( $x_{3}$ direction) and the wave vector $k_{\|}$(parallel to the interfaces). We choose $k_{\|}$along the $x_{1}$ direction without loss of generality. We consider a nonviscous fluid layer for which the viscous skin depth $\sigma=(2 \eta / \rho \omega)^{1 / 2}$ is much smaller than the fluid layer thickness $d_{f}$ over a very broad frequency range ( $\eta$ and $\rho$ are the viscosity and the density of the fluid). It is useful to know the surface elements of its elementary constituents, namely, the Green's function of an ideal fluid of thickness $d_{f}$, sound speed $v_{f}$, and mass density $\rho_{f}$ and an elastic isotropic solid characterized by its thickness $d_{s}$, longitudinal speed $v_{\ell}$, transverse speed $v_{t}$, and mass density $\rho_{s}$. In addition, the calculations of the transmission and reflection coefficients can be deduced only from the knowledge of the components of the Green's functions $g^{-1}(M M)$. Let us recall that the inverse Green's function of an ideal fluid layer in the space of the two surfaces $M_{f}=\left\{-d_{f} / 2,+d_{f} / 2\right\}$ of the layer is given by [60]

$$
\left[g_{f}\left(M_{f} M_{f}\right)\right]^{-1}=\left(\begin{array}{ll}
a_{f} & b_{f} \\
b_{f} & a_{f}
\end{array}\right),
$$

where

$$
\begin{aligned}
a_{f} & =-F \frac{C_{f}}{S_{f}}, \quad b_{f}=\frac{F}{S_{f}}, \\
C_{f} & =\cosh \left(\alpha_{f} d_{f}\right), \quad S_{f}=\sinh \left(\alpha_{f} d_{f}\right), \\
F & =\rho_{f} \frac{\omega^{2}}{\alpha_{f}} \text { and } \alpha_{f}=-j\left(\frac{\omega^{2}}{v_{f}^{2}}-k_{/ /}^{2}\right)^{\frac{1}{2}} .
\end{aligned}
$$

The inverse Green's function of the elastic solid layer in the space of the two surfaces $M_{s}=\left\{-d_{s} / 2,+d_{s} / 2\right\}$ of the layer is given by [60]

$$
\left[g_{s}\left(M_{s} M_{s}\right)\right]^{-1}=\left(\begin{array}{ll}
a_{s} & b_{s} \\
b_{s} & a_{s}
\end{array}\right)
$$

where

$$
\begin{aligned}
a_{s} & =-\gamma \frac{C_{\ell}}{S_{\ell}}-\beta \frac{C_{t}}{S_{t}}, \quad b_{s}=\frac{\gamma}{S_{\ell}}+\frac{\beta}{S_{t}}, \\
\gamma & =-\rho \frac{v_{t}^{4}}{\omega^{2} \alpha_{\ell}}\left(k_{\|}^{2}+\alpha_{t}^{2}\right)^{2}, \quad \beta=4 \rho \frac{v_{t}^{4}}{\omega^{2}} \alpha_{t} k_{\|}^{2}, \\
C_{t} & =\cosh \left(\alpha_{t} d_{s}\right), \quad C_{\ell}=\cosh \left(\alpha_{\ell} d_{s}\right), \\
S_{t} & =\sinh \left(\alpha_{t} d_{s}\right), \quad S_{\ell}=\sinh \left(\alpha_{\ell} d_{s}\right), \\
\text { and } \alpha_{t}^{2} & =k_{\|}^{2}-\frac{\omega^{2}}{v_{t}^{2}}, \quad \alpha_{\ell}^{2}=k_{\|}^{2}-\frac{\omega^{2}}{v_{\ell}^{2}} .
\end{aligned}
$$

The inverse Green's function of a semi-infinite fluid characterized by the longitudinal velocity of sound $v_{0}$ is given by $[60]$

$$
g_{f}^{-1}(0,0)=-F_{0},
$$

where $F_{0}$ is defined by Eq. (5c):

$$
F_{0}=\rho_{0} \frac{\omega^{2}}{\alpha_{0}} \text { and } \alpha_{0}=-j\left(\frac{\omega^{2}}{v_{0}^{2}}-k_{\|}^{2}\right)^{\frac{1}{2}} .
$$

It is worth noticing that the parameters $a_{f}, b_{f}$ [Eq. (5a)] and $a_{s}, b_{s}$ [Eq. (7a)] are real quantities, whereas $F_{0}$ [Eq. (9)] is a pure imaginary quantity for frequencies above the longitudinal sound line in the fluid. From the above results, we shall present analytical and numerical calculations of dispersion curves, transmission and reflection coefficients, phase, delay time, displacement field, and densities of states of acoustic waves in a given solid-fluid structure. In particular, we show in the next two sections the possibility to realize trapped modes as well as Fano and AIT resonances from single- and triple-layer structures. These resonances are obtained by combining appropriately the zeros of transmission (antiresonance) induced by the solid layers and the local resonances induced by either a single solid layer or a combined solid-fluid-solid layer with free surface boundary conditions. Let us mention that the zeros of transmission are the consequence of destructive interference of transverse and longitudinal waves inside the solid layers, leading to the cancellation of the transmitted waves $[52,61]$.

\section{SINGLE SOLID LAYER}

Even though the problem of a single solid layer inserted between two semi-infinite fluids has been the subject of several studies [62], however, to our knowledge the possibility of existence of trapped (or BIC) modes and Fano resonances in such systems has not been treated before. In this section, we will show that a single solid layer inserted between two fluids may exhibit such modes. We first present the analytic demonstration and then give practical illustrations. The reader not directly interested by the demonstrations may skip the first part and go directly to the illustrations. 


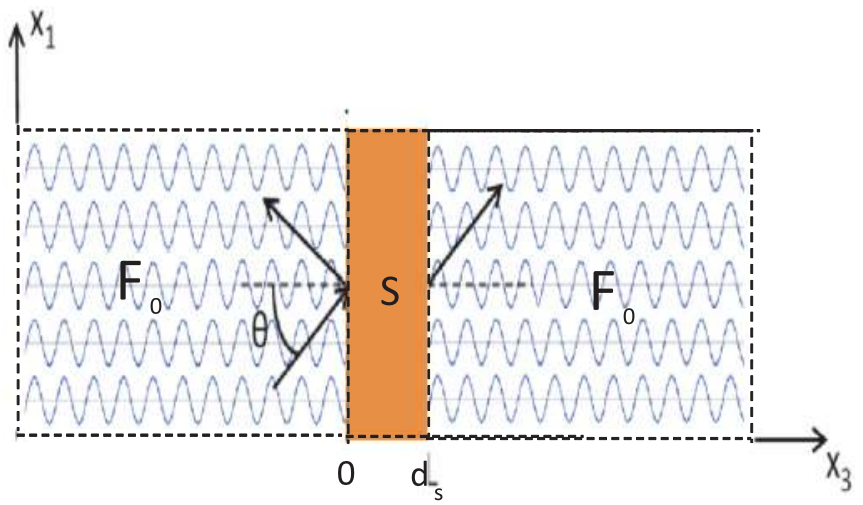

FIG. 1. Schematic representation of a solid layer of thickness $d_{s}$ sandwiched between two semi-infinite fluids $F_{0} . \theta$ is the angle of incidence.

\section{A. Analytical results}

Consider a single solid layer of thickness $d_{s}$ inserted between two semi-infinite fluids (Fig. 1). The inverse Green's function $g(M M)^{-1}$ of the whole system in the space of interfaces $M \equiv\left\{0, d_{s}\right\}$ can be obtained as a linear superposition of the matrix [Eq. (6)] describing the solid layer and the scalar [Eq. (8)] describing the surrounding fluids, namely,

$$
\left[g_{s}\left(M_{s} M_{s}\right)\right]^{-1}=\left(\begin{array}{cc}
a_{s}-F_{0} & b_{s} \\
b_{s} & a_{s}-F_{0}
\end{array}\right) .
$$

An incident plane wave launched from the left (Fig. 1) gives rise to reflection and transmission waves. The expressions giving these two quantities can be obtained from Eqs. (3) and (10) as [51]

$$
r=1+2 F_{0} g(0,0)=\frac{a_{s}^{2}-b_{s}^{2}-F_{0}^{2}}{\left(a_{s}-b_{s}-F_{0}\right)\left(a_{s}+b_{s}+F_{0}\right)}
$$

and

$$
t=2 F_{0} g\left(0, d_{s}\right)=\frac{2 F_{0} b_{s}}{\left(a_{s}-b_{s}-F_{0}\right)\left(a_{s}+b_{s}+F_{0}\right)} .
$$

In what follows, we shall concentrate on the transmission coefficient. From Eqs. (7a) and (7b), the expression of $t$ [Eq. (12)] can be written explicitly as

$t=\frac{F_{0}\left(\gamma S_{t}^{\prime} C_{t}^{\prime}+\beta S_{l}^{\prime} C_{l}^{\prime}\right)}{\left[\gamma C_{l} S_{t}^{\prime}+\beta S_{l}^{\prime} C_{t}^{\prime}+F_{0} S_{l}^{\prime} S_{t}^{\prime}\right]\left[\gamma C_{t}^{\prime} S_{l}^{\prime}+\beta S_{t}^{\prime} C_{l}^{\prime}+F_{0} C_{l}^{\prime} C_{t}^{\prime}\right]}$,

where $C_{t}^{\prime}=\cosh \left(\alpha_{t} d_{s} / 2\right), C_{\ell}^{\prime}=\cosh \left(\alpha_{\ell} d_{s} / 2\right), S_{t}^{\prime}=\sinh \left(\alpha_{t}\right.$ $\left.d_{s} / 2\right)$, and $S_{\ell}^{\prime}=\sinh \left(\alpha_{\ell} d_{s} / 2\right)$.

It is clear from Eq. (13) that the transmission zeros are given by

$$
\gamma S_{t}^{\prime} C_{t}^{\prime}+\beta S_{l}^{\prime} C_{l}^{\prime}=0
$$

Also, the symmetrical and antisymmetrical modes of the system are given by the zeros of the denominator of $t$ [Eq. (13)], namely,

$$
\gamma C_{l} S_{t}^{\prime}+\beta S_{l}^{\prime} C_{t}^{\prime}+F_{0} S_{l}^{\prime} S_{t}^{\prime}=0
$$

and

$$
\gamma C_{t}^{\prime} S_{l}^{\prime}+\beta S_{t}^{\prime} C_{l}^{\prime}+F_{0} C_{l}^{\prime} C_{t}^{\prime}=0
$$

The left-hand sides of Eqs. (15) and (16) are complex quantities. Poles of $t$ [Eq. (13)] or equivalently the Green's functions [Eqs. (11) and (12)] refer either to resonances or to bound states. The imaginary parts in Eqs. (15) and (16) are responsible of the broadening of resonances in the transmission coefficient [Eq. (13)]. The real parts give the Lamb modes of the solid layer of thickness $d_{s}$ with free stress surface boundary conditions. However, if real and imaginary parts of Eqs. (15) and (16) vanish at the same frequency, then we get trapped modes (bound states), i.e., resonances with zero width falling in the continuum, the so-called bound in continuum (BIC) states. One can notice that Eqs. (15) and (16) lead, respectively, to the following simple equations:

$$
S_{t}^{\prime}=0 \text { and } S_{l}^{\prime}=0
$$

and

$$
C_{t}^{\prime}=0 \text { and } C_{l}^{\prime}=0,
$$

giving the poles of the Green's function or equivalently the transmission and reflection coefficients [Eqs. (11)-(13)], and thereby the BIC modes. These modes are independent of the nature of the fluids surrounding the solid layer. The above conditions [Eqs. (18) and (17)] occur for

$$
\omega=v_{t} \sqrt{k_{\|}^{2}+\left(\frac{m \pi}{d_{s}}\right)^{2}}=v_{l} \sqrt{k_{\|}^{2}+\left(\frac{n \pi}{d_{s}}\right)^{2}},
$$

which gives the coordinates of the angular frequency $\omega_{m, n}$ and the wave number $k_{\|}^{m, n}$, respectively, as

$$
\omega_{m, n}=\frac{v_{l}}{\sqrt{\left(\frac{v_{l}}{v_{t}}\right)^{2}-1}} \frac{\pi}{d_{s}} \sqrt{m^{2}-n^{2}}
$$

and

$$
k_{\|}^{m, n}=\frac{\pi}{d_{s}} \sqrt{\frac{m^{2}-n^{2}\left(\frac{v_{l}}{v_{t}}\right)^{2}}{\left(\frac{v_{l}}{v_{t}}\right)^{2}-1}},
$$

where $m$ and $n$ are both odd or even integers. Let us mention that the incidence angle $\theta$ (Fig. 1) is related to the parallel wave vector $k_{\|}$by the expression $k_{\|}=\frac{\omega}{v_{0}} \sin (\theta)$.

Equations (20) and (21) are well known as the coordinates of the crossing points of the uncoupled dilatation and shear modes of the solid layer [63]. The corresponding incidence angles are given by

$$
\sin (\theta)=\frac{v_{0}}{v_{l}}\left(\frac{m^{2}-n^{2}\left(\frac{v_{l}}{v_{t}}\right)^{2}}{m^{2}-n^{2}}\right)
$$

It is worth noticing that when Eqs. (17) and (18) hold, the numerator and denominator of the transmission coefficient [Eq. (13)] vanish, however, one can show after some tedious calculations (not given here) that a Taylor expansion of $t$ around $\omega_{m, n}$ and $k_{\|}^{m, n}$ gives well-defined values of $t$. In particular, we obtained a complete transmission rate $T=|t|^{2}=1$ for any combination $(m, n \neq 0)$ and $T<1$ for the pair $(m, n=0)$ (see below).

Another interesting quantity that can be deduced from the Green's function (10) is the density of states (DOS), especially 
TABLE I. Velocities of sound and mass densities of Plexiglas, PMMA, alcohol, and water.

\begin{tabular}{lccc}
\hline \hline & $\rho\left(\mathrm{Kg} / \mathrm{m}^{3}\right)$ & $v_{t}\left(10^{3} \mathrm{~m} / \mathrm{s}\right)$ & $v_{\ell}\left(10^{3} \mathrm{~m} / \mathrm{s}\right)$ \\
\hline Plexiglas & 1200 & 1.38 & 2.7 \\
PMMA & 1190 & 1.4 & 2.8 \\
Alcohol & 786.51 & & 1.103 \\
Water & 1000 & & 1.49 \\
\hline \hline
\end{tabular}

one can determine the variation of the density of states $\Delta n$ between the structure in Fig. 1 and a reference system formed out of the same volumes of the decoupled semi-infinite fluids and the solid layer. This quantity is given by [64]

$$
\Delta n(\omega)=\frac{1}{\pi} \frac{d}{d \omega} \operatorname{Arg} \operatorname{det}\left\{\frac{g\left(M_{0} M_{0}\right)}{\left[g_{f}(0,0) g_{f}\left(d_{s}, d_{s}\right)\right]^{1 / 2}}\right\},
$$

where $g\left(M_{0} M_{0}\right)$ is the Green's function of the whole system at its both extremities $M_{0}=\left\{0, d_{s}\right\}$, whereas $g_{f}(0,0)$ and $g_{f}\left(d_{s}, d_{s}\right)$ are the elements of the Green's functions at the surfaces 0 and $d_{s}$ of the two semi-infinite fluids. In Eq. (23), we have subtracted the discrete modes of the finite solid medium [65].

\section{B. Numerical illustrations}

Consider, for example, the case of a Plexiglas layer of thickness $d_{s}$ inserted in water. Table I gives the numerical values of velocities of sound and mass densities of the materials used in the calculation.

It is well established [52] that when a solid layer is inserted between two fluids, there exists a critical angle $\theta_{c}$ above which the transmission never reaches zero. In the case of Plexiglas solid layer immersed in water $\theta_{c}=39^{\circ}$. Figure 2 gives the variation of the transmission amplitude (with color scale) versus the dimensionless frequency $\Omega=\frac{\omega d_{s}}{v_{0}}$ and $\theta$.

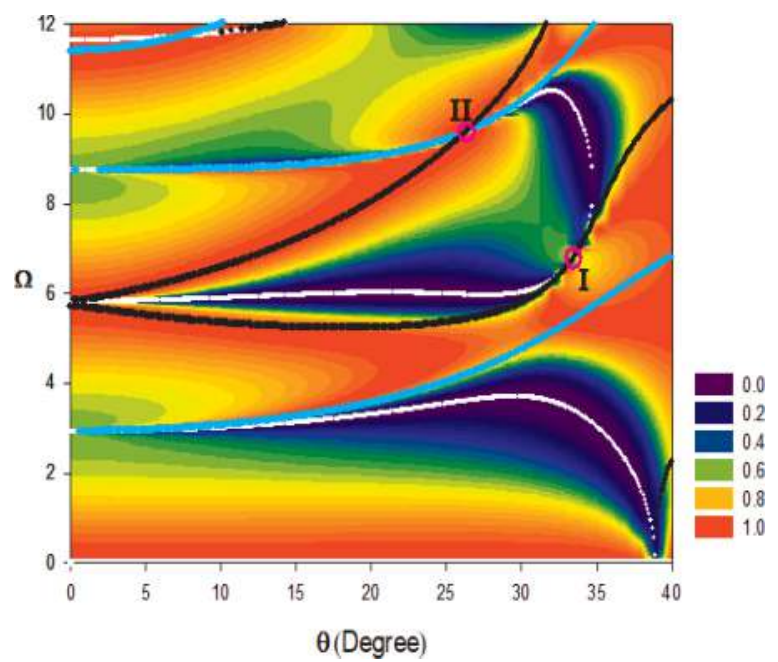

FIG. 2. Transmission coefficient (in color scale) versus the dimensionless frequency $\Omega$ and the angle of incidence $\theta$. White dotted curves represent the frequencies of the transmission zeros (total reflection), whereas black and cyan curves are associated to symmetric and antisymmetric modes of the free surface solid layer, respectively.
The white dots give the positions of the transmission zeros [Eq. (14)]. As mentioned above, these transmission zeros occur for $\theta<\theta_{c} \simeq 39^{\circ}$. The circles labeled I and II represent BIC modes; they are solutions of Eqs. (20) and (22) for $(n=0, m=2)$ and $(n=1, m=3)$, respectively. The corresponding coordinates are, respectively, $\left(\theta_{I}=33.49^{\circ}, \Omega_{I}=\right.$ $6.77)$ and $\left(\theta_{I I}=26.34^{\circ}, \Omega_{I I}=9.5\right)$. Black and cyan curves correspond, respectively, to symmetric and antisymmetric modes of the solid layer with free stress surfaces (i.e., Lamb modes). As it is demonstrated in Sec. III A, BIC modes can be also understood as the intersection of Lamb modes of the solid layer with the transmission zeros as it is the case around points I and II. Now, when the solid layer (Plexiglas) is inserted in water, the Lamb modes will become resonances, however, because of the small impedance of water in comparison with Plexiglass layer, the positions of the resonances become close to those of the Lamb modes, and when the latter fall near to the transmission zeros, they will give rise to Fano resonances (see below).

In order to show the behavior of the trapped modes and Fano resonances in the transmission amplitude around $\theta=\theta_{I}=$ $33.49^{\circ}$ and $\Omega=\Omega_{I}=6.77$, we have plotted in Figs. 3(a)-3(c) the transmission versus $\Omega$ for $\theta=33.2^{\circ}<\theta_{I}, \theta=\theta_{I}$, and $\theta=33.8^{\circ}>\theta_{I}$, respectively. The filled circles on the abscissa of Figs. 3(b), 3(e), and 3(h) indicate the position of the trapped mode. Figures 3(a) and 3(c) clearly show the existence of a Fano-type resonance around $\Omega_{I}$ for $\theta<\theta_{I}$ [Fig. 3(a)] and $\theta>\theta_{I}$ [Fig. 3(c)], respectively. This resonance is characterized by an asymmetric line shape (i.e., a resonance close to an antiresonance). The Fano resonance disappears (collapses) for $\theta=\theta_{I}$ [Fig. 3(b)] giving rise to a zero width resonance. This is a characteristic of a trapped mode, i.e., a discrete mode falling in the continuum modes (radiating modes) of the surrounding fluids. As indicated in Sec. III A, when the resonance collapses, the transmission rate $T$ reaches a constant value lower than unity $[T \simeq 0.6$, Fig. 3(b)]. Ladron de Guevara et al. [66] called these hidden resonances "ghost Fano resonance" as they collapse in the transmission amplitude. Similar results have been found by Voo and Chu [67] for the electron ballistic transport through a mesoscopic two-lead ring and Shipman and Venakides [68] for electromagnetic waves in periodically structured slabs in the presence of nonrobust guided slab modes.

Figures 3(d)-3(f) give the variation of the phase of the transmission for the same values of $\theta$ as in Figs. 3(a)-3(c), respectively. Figures 3(d) and 3(f) clearly show a noticeable increasing of the phase by an amount of $+\pi$ around the position of the Fano resonance followed by a phase drop of $-\pi$ due to the transmission zero. However, when the Fano resonance and the transmission zero fall at the same frequency [see Fig. 3(e) around $\left.\Omega_{I}=6.77\right]$, then we obtain a cancellation of both phases leading to a monotonic increasing of the phase. To characterize better the Fano and trapped modes, we have plotted in Figs. 3(g), 3(h), and 3(k), the DOS versus $\Omega$ for the same values of $\theta$ as in Figs. 3(a), 3(b), and 3(c), respectively. Figures $3(\mathrm{~g})$ and $3(\mathrm{k})$ clearly show the existence of a very thin resonance around $\Omega_{I}=6.77$, its width decreases as far as $\theta$ tend to $\theta_{I}$ and collapses for $\theta=\theta_{I}$. However, the other resonances (around $\Omega=5.5$ and 10.5) exhibit broad linewidths for all values of $\theta$ around $\theta_{I}$. 

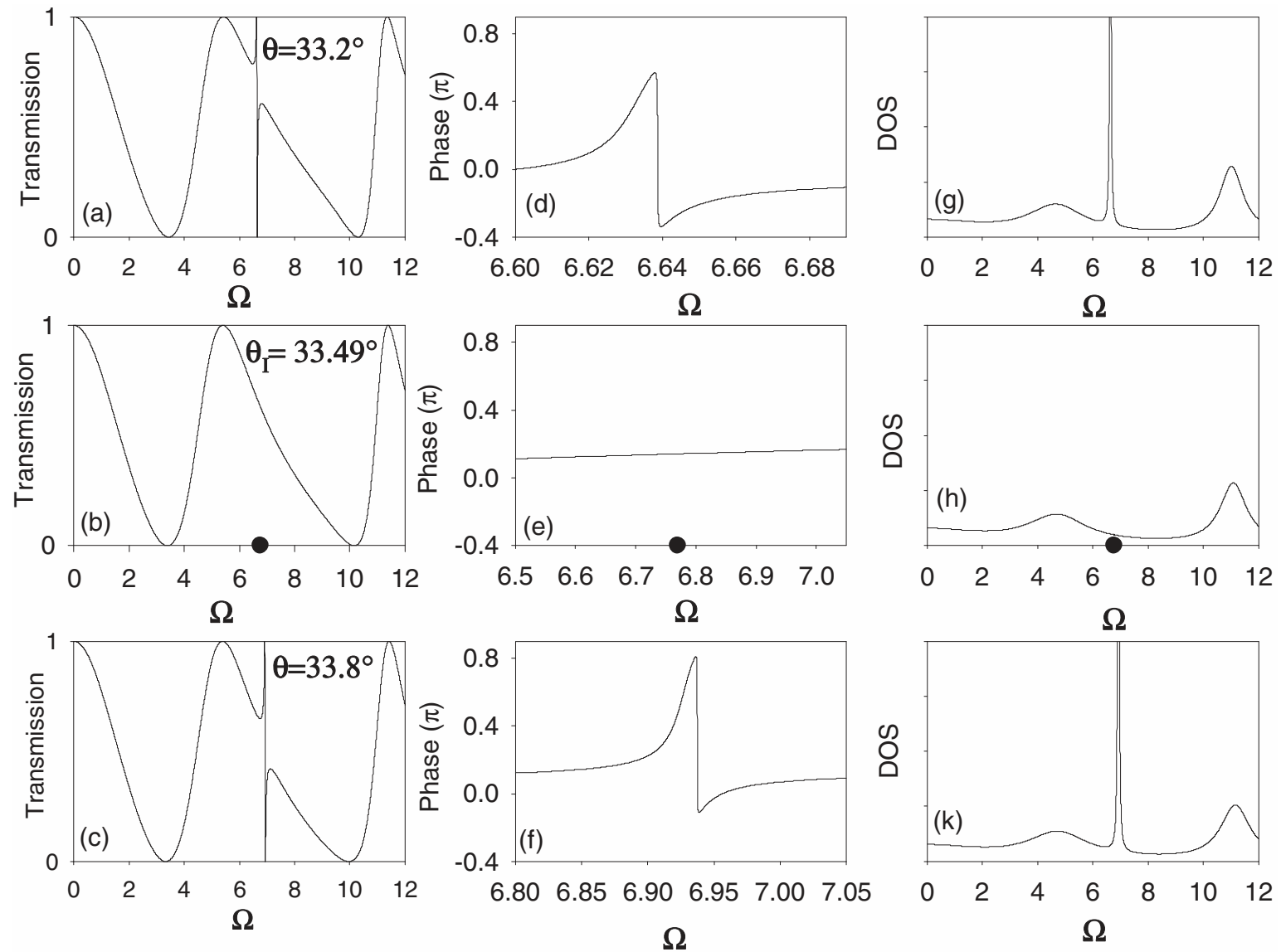

FIG. 3. Variation of the transmission coefficient (a), phase (d), and DOS (in arb. units) (g) versus the dimensionless frequency $\Omega=\frac{\omega d_{s}}{v_{0}}$ for the structure depicted in Fig. 1 for $\theta=33.2<\theta_{I}=33.49^{\circ}$. The phase is represented in the restricted frequency domain around $\Omega_{I}=6.77$. (b), (e), (h) Represent the same curves as (a), (d), (g) but for $\theta=\theta_{I}=33.49^{\circ}$. (c), (f), (k) Represent the same curves as (a), (d), (g) but for $\theta=33.8>\theta_{I}=33.49^{\circ}$.

In the above illustrations, we have neglected the dissipation effects. However, acoustic absorption in the constituting materials may affect the shape of the Fano (and later AIT) resonances depending on the material properties and frequency range under consideration. We give a discussion of the dissipation effects in the Appendix. Qualitatively speaking, the acoustic absorption will broaden the narrow resonances, and at high dissipation, the peaks may even lose their Fano shape. So, to observe the Fano resonance behavior, it would be necessary either to work with materials with very low acoustic absorption or choose the angle of observation a little shifted with respect to the BIC states to deal with broader resonant effect.

Another example of the existence of Fano and trapped modes for $n \neq 0$ is given in Figs. 4(a)-4(c) for $\theta_{I I}=26.34^{\circ}$ and $\Omega=9.5$. These solutions correspond to $n=1$ and $m=3$ in Eqs. (18) and (17). Figures 4(a) and 4(c) clearly show the existence of an asymmetric Fano line-shape resonance for $\theta=24^{\circ}$ and $27.5^{\circ}$, respectively. These resonances collapse for $\theta=26.34^{\circ}$ giving rise to a trapped mode. As mentioned above, one can show analytically using Taylor expansion around $\Omega=9.5$ that the transmission reaches unity where the Fano resonance disappears. These results are confirmed also in the DOS spectra [Figs. 4(d)-4(f)] where the Fano resonances at $\theta=24^{\circ}$ [Fig. 4(d)] and $\theta=27.5^{\circ}$ [Fig. 4(f)] collapse at $\theta=26.34^{\circ}$, giving rise to a BIC mode.

\section{SOLID-FLUID-SOLID TRIPLE LAYER}

In this section we consider a triple layer composed of alternate solid-fluid-solid layers inserted between two semiinfinite fluids (Fig. 5). In general, the nature of the two solids and the fluids can be similar or different from each other. This structure exhibits more degrees of freedom in comparison with the single solid layer. In particular, we show that in addition to the Fano resonances displayed by one solid layer (Sec. III), there are other types of Fano and AIT resonances that can be induced by the new structure.

\section{A. Fano resonance}

As mentioned above, the Fano resonance is characterized by an asymmetric line shape where a resonance falls near an antiresonance (or a transmission zero) in the transmission spectrum. As indicated in Sec. III, the transmission zeros are induced by the solid layer inserted between two fluids and are a characteristic of the solid layer which plays the role of a resonator. Therefore, we shall first consider the case where the solid layers are identical, i.e., a symmetric triple layer (Fig. 5 with $S_{1}$ identical to $S_{2}$ ) in order to get the transmission zeros induced by both solid layers at the same frequency. 

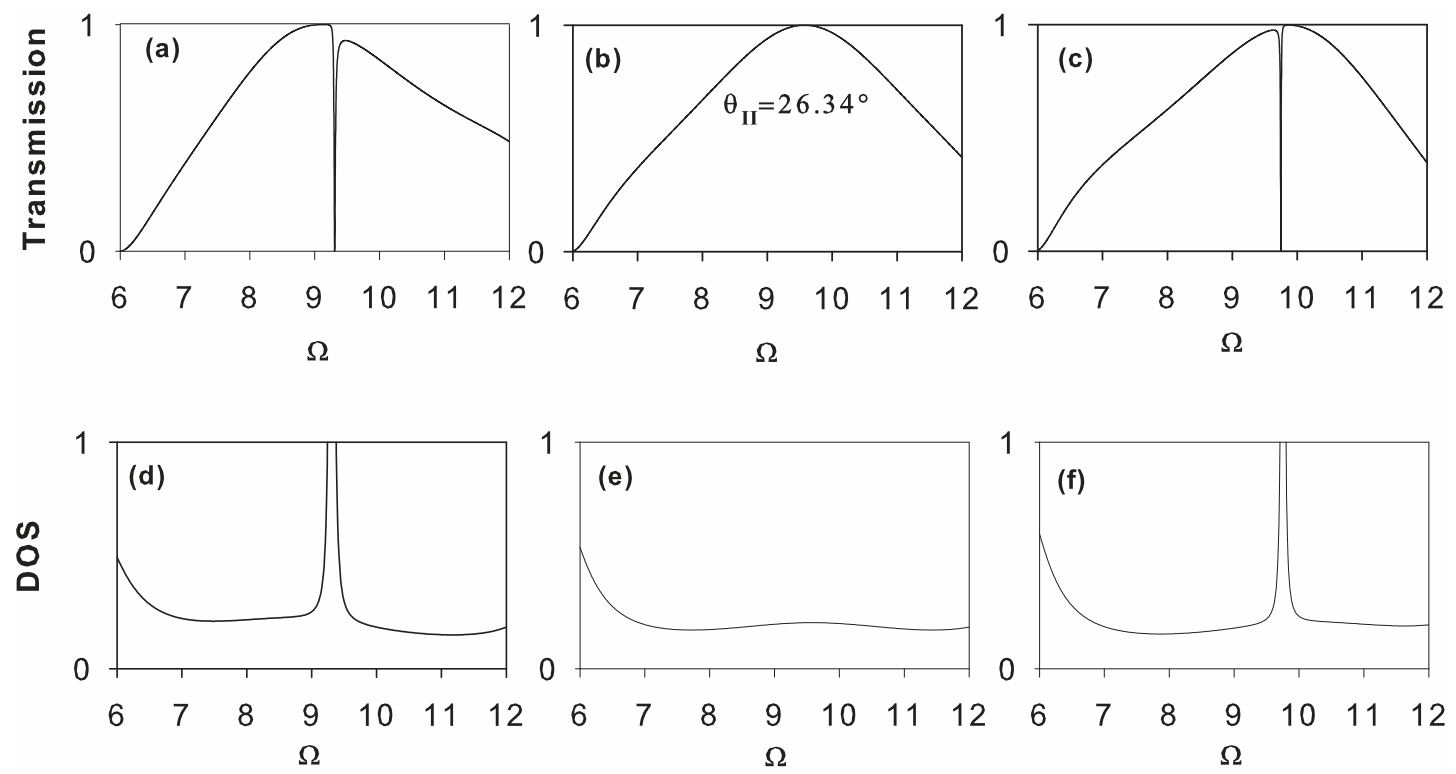

FIG. 4. (a)-(c) Variation of the transmission coefficient versus the dimensionless frequency $\Omega=\frac{\omega d_{s}}{v_{0}}$ for the structure depicted in Fig. 1 for $\theta=24^{\circ}<\theta_{I I}=26.34^{\circ}, \theta=\theta_{I I}$, and $\theta=27.5^{\circ}>\theta_{I I}=26.34^{\circ}$, respectively. (d)-(f) Characterize the DOS (in arb. units) versus $\Omega$ for the same values of $\theta$ as in Figs. 3(a)-3(c), respectively.

Figure 5 shows the schematic representation of the triple layer made of a fluid layer $F$ of thickness $d_{f}$ sandwiched by two solid layers $S_{1}$ and $S_{2}$ of thicknesses $d_{s 1}$ and $d_{s 2}$, respectively. The whole system is inserted between two fluids $F_{0}$. As mentioned above, in order to realize a Fano resonance, we should consider the solid layers identical (i.e., $d_{s 1}=d_{s 2}=d_{s}$ ). In order to calculate the transmission and reflection coefficients using the Green's function method, we need to construct first the inverse Green's function $[g(M M)]^{-1}$ of the whole system in the space of interfaces $M \equiv\left\{0, d_{s}, d_{s}+d_{f}, 2 d_{s}+\right.$ $\left.d_{f}\right\}$. This matrix is obtained as a linear superposition of the matrices given by Eqs. (4) and (6), namely,

$$
[g(M M)]^{-1}=\left(\begin{array}{cccc}
a_{s}-F_{0} & b_{s} & 0 & 0 \\
b_{s} & a_{s}+a_{f} & b_{f} & 0 \\
0 & b_{f} & a_{f}+a_{s} & B_{s} \\
0 & 0 & b_{s} & a_{s}-F_{0}
\end{array}\right) .
$$

An incident plane wave launched from the left (Fig. 5) gives rise to reflection and transmission waves. The expressions giving these two quantities can be obtained as in Sec. III, namely,

$$
r=\frac{\left[\left(a_{s}+a_{f}\right)\left(a_{s}-F_{0}\right)-b_{s}^{2}\right]\left[\left(a_{s}+a_{f}\right)\left(a_{s}+F_{0}\right)-b_{s}^{2}\right]-b_{f}^{2}\left(a_{s}-F_{0}\right)\left(a_{s}+F_{0}\right)}{\Delta}
$$

and

$$
t=2 F_{0} \frac{b_{f} b_{s}^{2}}{\Delta}
$$

where

$$
\begin{aligned}
\Delta= & \operatorname{det}\left[g^{-1}(M M)\right]=\left[\left(a_{s}-F_{0}\right)\left(a_{s}+a_{f}-b_{f}\right)-b_{s}^{2}\right] \\
& \times\left[\left(a_{s}-F_{0}\right)\left(a_{s}+a_{f}+b_{f}\right)-b_{s}^{2}\right]
\end{aligned}
$$

is the determinant of the matrix $g^{-1}(M M)$ given in Eq. (24). The eigenmodes of the whole system are given by Eq. (2) or, equivalently,

$$
\Delta=0 .
$$

Equation (26) clearly shows that the transmission zeros are given by

$$
b_{s}=0,
$$

where $b_{s}$ is given by Eq. (7a). In addition, the eigenmodes of the triple layer with free boundary conditions are given by Eqs. (27) and (28) by taking $F_{0}=0$, namely,

$$
\left[a_{s}\left(a_{s}+a_{f}-b_{f}\right)-b s^{2}\right]\left[a_{s}\left(a_{s}+a_{f}+b_{f}\right)-b s^{2}\right]=0 .
$$

Therefore, if Eqs. (29) and (30) hold simultaneously, then Eq. (27) vanishes $(\Delta=0)$, leading to the eigenmodes of the triple layer independently of the nature of the surrounding fluids (i.e., $F_{0}$ ). These modes can be qualified as BIC modes as they represent eigenmodes falling in the continuum of the surrounding fluids without radiating their energy. Also, these modes do not contribute to the transmission [Eq. (26)] and are characterized by an infinite lifetime as the full width at half maximum (FWHM) of these resonances vanishes (see below).

Consider, for example, the case of a water layer inserted between two identical Plexiglas layers. The thicknesses of all 


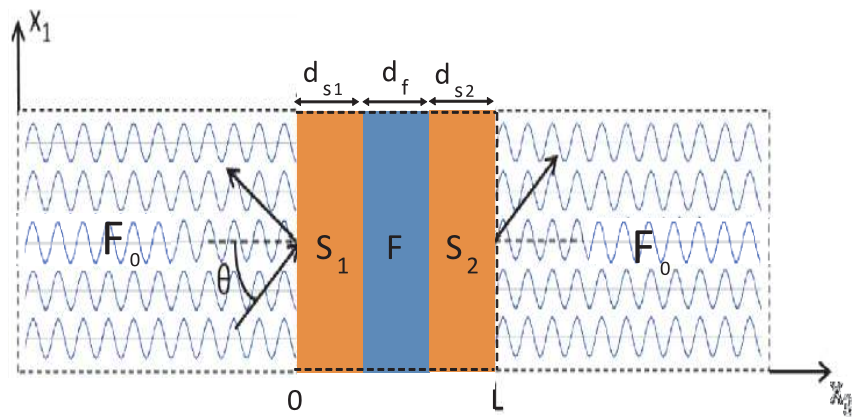

FIG. 5. Schematic representation of a triple-layer structure made of a fluid layer $F$ of thickness $d_{f}$ sandwiched between two solids $S_{1}$ and $S_{2}$ of thicknesses $d_{s 1}$ and $d_{s 2}$, respectively. The whole system is inserted between two fluids $F_{0} . \theta$ is the angle of incidence and $L=d_{f}+d_{s 1}+d_{s 2}$ is the total length of the triple layer.

the layers are supposed to be the same $d_{s 1}=d_{s 2}=d_{s}=d_{f}$ and the whole layers are immersed in water. The solutions of Eqs. (29) and (30) for the incidence angle $\theta$ less than the critical angle $\theta_{c}$ and for the dimensionless frequency $\Omega=\frac{\omega d_{f}}{v_{f}}<$ 4 give the two following trapped modes: $\left(\theta=32.6^{\circ}, \Omega=\right.$ $3.51)$ and $\left(\theta=37.6^{\circ}, \Omega=1.85\right)$. In the following, we shall
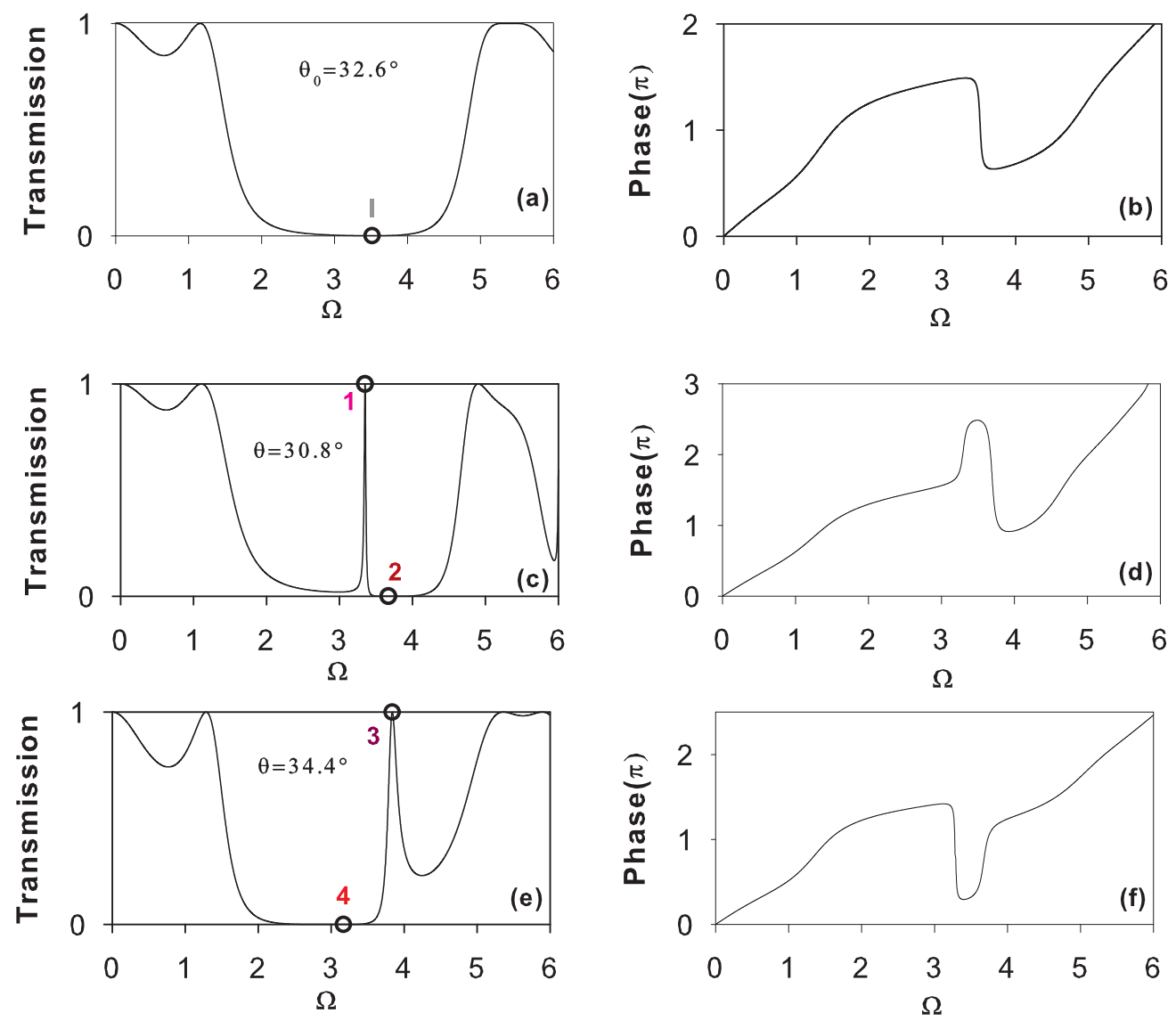

FIG. 6. (a) Variation of the transmission coefficient versus the dimensionless frequency $\Omega=\frac{\omega d_{f}}{v_{f}}$ for the structure depicted in Fig. 5. The width of the layers is taken such as $d_{s}=d_{f}$. The modes $I$ at $\Omega=\Omega_{0}=3.52$ correspond to the trapped mode where two transmission zeros induced by the two solid layers coincide with the hidden Fano resonance. (b) The same as (a) but for the variation of the phase. (c), (e) The same as (a) but for $\theta=30.8^{\circ}$ and $34.4^{\circ}$ with their phases (d) and (f), respectively. Modes 1 and 3 correspond to Fano resonances, whereas modes 2 and 4 correspond to the transmission zeros. 


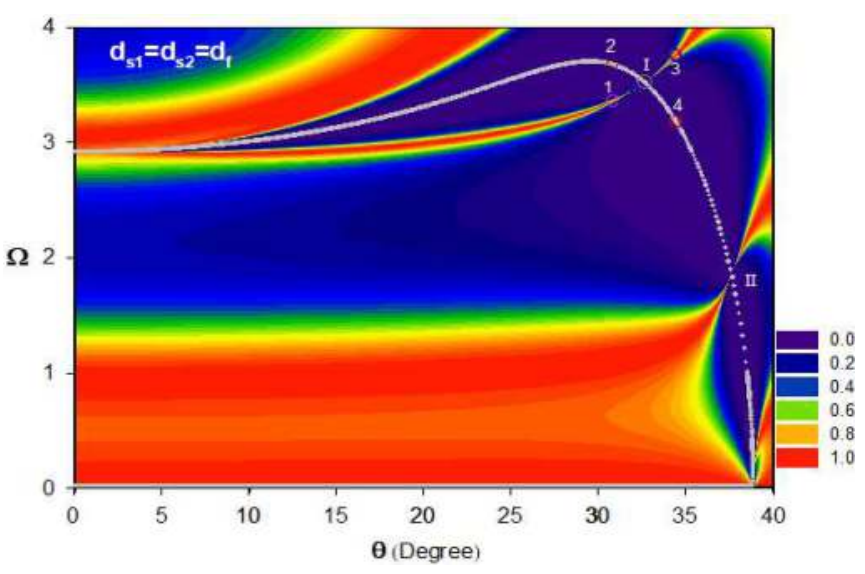

FIG. 7. Transmission coefficient versus the dimensionless frequency $\Omega$ and the angle of incidence $\theta$. Color areas indicate the magnitude of the transmission rate. White dotted curves represent the frequencies of the transmission zeros (total reflection).

for $\theta=\theta_{0}$. A discussion about the effect of dissipation is given in the Appendix.

In order to get a better insight about the behavior of the Fano resonance as a function of the incidence angle $\theta$ and the frequency $\Omega$, we plotted in Fig. 7 the variation of the transmission amplitude (with color scale) versus $\Omega$ and $\theta$. The white dots give the positions of the transmission zeros [Eq. (29)]. As mentioned above, these transmission zeros occur for $\theta<\theta_{c} \simeq 39^{\circ}$. Around $\left(\theta=32.6^{\circ}, \Omega=3.51\right)$ and $(\theta=$ $37.6^{\circ}, \Omega_{0}=1.85$ ), one can notice particular points where the maxima of transmission (red areas) become very thin before crossing transmission zeros and giving rise to trapped modes (i.e., resonances with zero width) labeled I and II. As mentioned above, these modes are given by Eqs. (29) and (30). Around $\theta=32.6^{\circ}$, the resonance line (red curve) falls below (above) the transmission zero curve as it is illustrated in Fig. 7. The modes labeled 1, 2, 3, and 4 in Figs. 6(c) and 6(e) are indicated in Fig. 7 around the crossing point I where the resonance and the antiresonance fall at the same frequency. The same behavior occurs for the trapped mode II around $\theta=37.6^{\circ}$ and $\Omega=1.85$. It is worth noticing that other trapped modes like those discussed in Fig. 2 above $\Omega=4$ exist also in the triple-layer structure as these modes are an intrinsic property of solid layers. We have avoided discussing these modes in Fig. 7.
The Fano resonance depends on the nature of the fluid inserted between the solid layers. We have checked the case where we have replaced water layer by alcohol layer (Table I), keeping the other parameters the same as in Fig. 7. We have found almost the same results as in Fig. 7, but the Fano resonance falls at lower frequencies and becomes slightly larger.

In order to show the stopping and filtering of the waves by the triple layer, we have plotted in Fig. 8 the spatial localization of the displacement field for the modes labeled I, 1, and 2 in Figs. 6(a), 6(c), and 7. Figure 8(a) gives the displacement field for the mode labeled 2 [Fig. 6(c)] which is associated to the transmission zero. As predicted, this mode is blocked by the first solid layer it encounters. However, the mode labeled 1 [Fig. 6(c)] associated to the Fano resonance remains well confined in the triple layer, especially in the central fluid layer, and continues its propagation in the fluid at the right side. As concerns the mode labeled I [Fig. 6(a)] associated to a BIC mode, it is well confined in the triple layer and vanishes at its both extremities [Fig. 8(c)]. Even though this mode can be excited from the fluid at the left side, it behaves as a stationary mode without interacting with the surrounding semi-infinite fluids. Indeed, the incident wave launched in the fluid at the left side of the system [Fig. 8(c)] does not propagate at the right side and remains confined in the triple layer.

In order to show that the resonances in Figs. 6(c) and 6(e) are of Fano type with an asymmetric shape, the latter should follow the Fano formula, namely [3],

$$
T=A \frac{\left(\omega-\omega_{R}+q \Gamma\right)^{2}}{\left(\omega-\omega_{R}\right)^{2}+\Gamma^{2}},
$$

where $A=\frac{1}{q^{2}}, \omega_{R}$, and $\Gamma$ are the standard parameters that denote the position and the width of the resonance, respectively. $q$ is the so-called Fano parameter [2] which describes the degree of asymmetry of the resonance. Figure 9 gives a comparison between the exact results of the transmission coefficient (solid curve) and the curve obtained for Eq. (31) (open circle) for $\theta=32.6^{\circ}$. A good fit is obtained with the following parameters $q=10.763$ and $\Gamma=0.038$ (in units of $\left.d_{f} / v_{f}\right)$.

In order to get a better insight about the dependence of the Fano parameter $q$ and the width $\Gamma$ or equivalently the quality factor $Q=\frac{\Omega_{R}}{\Gamma}$ of the Fano resonance upon the incidence angle $\theta$, we plotted in Figs. 10(b) and 10(c) the variation of $q$ and

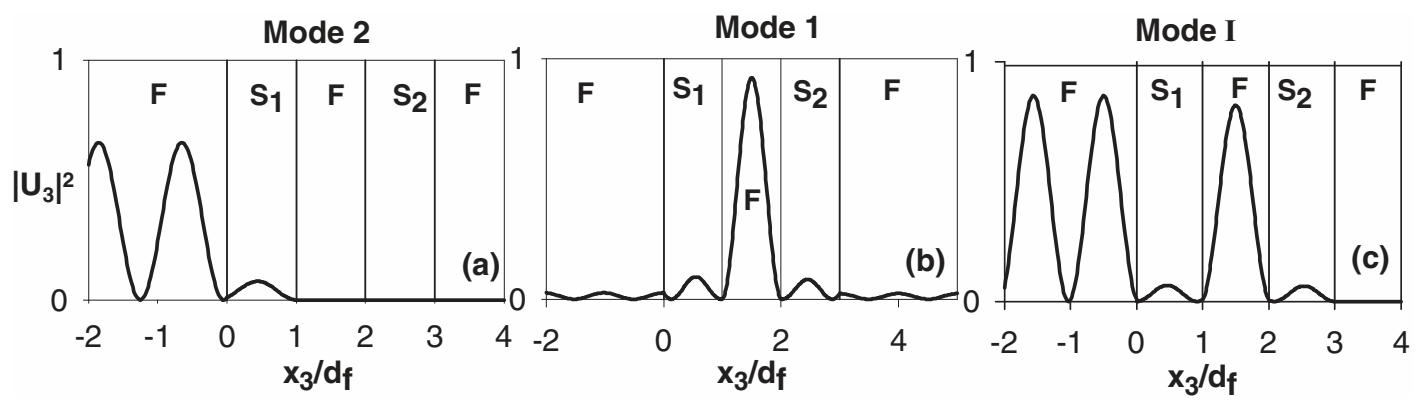

FIG. 8. (a) Square modulus of the displacement field versus the space position for the mode labeled 2 (transmission zero) in Figs. 6(c) and 7. (b) The same as in (a) but for the mode labeled 1 (Fano resonance) in Figs. 6(c) and 7. (c) The same as in (a) but for the mode labeled I (BIC mode) in Figs. 6(a) and 7. 


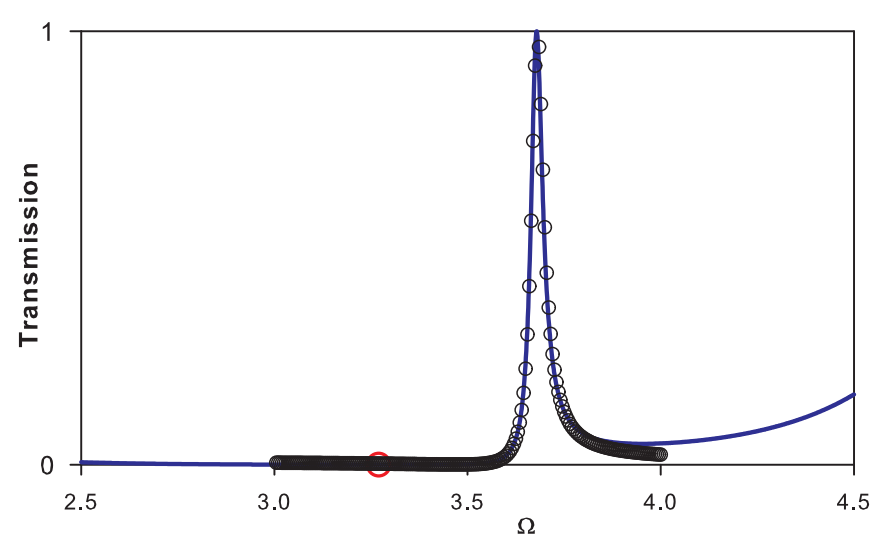

FIG. 9. Comparison between the transmission curves associated to exact results (solid line) and fitted results (open circles) obtained from Eq. (31) versus the dimensionless frequency $\Omega$ for $d_{s}=d_{f}$ and $\theta=32.6^{\circ}$.

$Q$ versus $\theta$, for $\theta$ close to $\theta_{0}=32.6^{\circ}$. Figure 10(a) recalls the dispersion curves of the Fano resonance (dashed curves) and the transmission zero (full curve) versus $\theta$ as in Fig. 7. At the crossing point I, the Fano parameter $q$ [Fig. 10(b)] which is responsible of the asymmetric Fano profile of the resonance diverges and changes sign around $\theta_{0}=32.6^{\circ}$. Also, the quality factor $Q$ [Fig. 10(c)] diverges around $\theta_{0}$ as the width of the Fano resonance vanishes at the incidence angle $\theta_{0}$.

\section{B. AIT resonance}

Unlike the asymmetric Fano resonance, in order to show the possibility of existence of the AIT resonance (symmetric Fano resonance), it is sufficient to take the thicknesses of the two solid layers $d_{s_{1}}$ and $d_{s_{2}}$ (Fig. 5) slightly different from each other for a particular angle of incidence. Our goal consists to squeeze a resonance between two transmission zeros induced by the two solid layers. In what follows, we shall give the transmission and reflection coefficients for an asymmetric triple-layer structure. The inverse of the Green's function $g^{-1}(M M)$ in the interface space $M=\left\{0, d_{s 1}, d_{s 1}+d_{f}, d_{s 1}+d_{s 2}+d_{f}\right\}$ of the whole system is obtained as a juxtaposition of the three different layers and the surrounding semi-infinite fluids. The parameters of the two different solid layers are given by Eq. (7) where we should add subscripts 1 and 2 in order to distinguish the

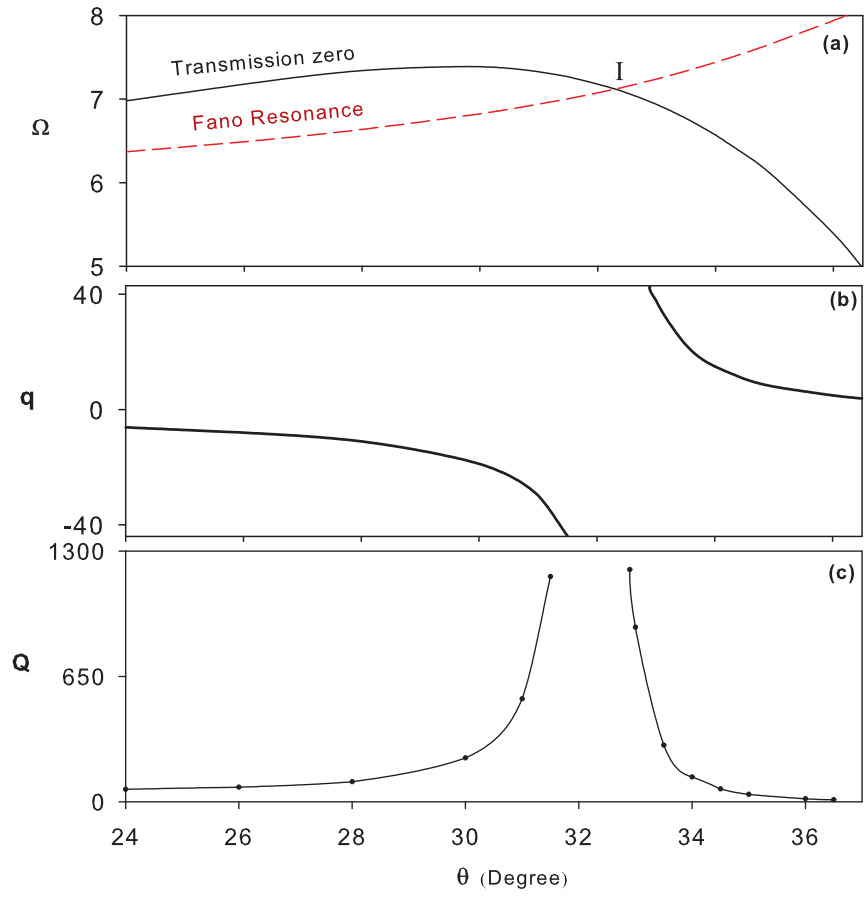

FIG. 10. (a) Variation of the dimensionless frequency $\Omega$ of the Fano resonance and the transmission zero versus $\theta$ as in Fig. 7 . (b), (c) Variation of the Fano parameter $q$ and the quality factor $Q$ versus $\theta$.

left and right solid-layer parameters, respectively. Therefore, $g^{-1}(M M)$ is given by

$$
[g(M M)]^{-1}=\left(\begin{array}{cccc}
a_{s_{1}}-F_{0} & b_{s_{1}} & 0 & 0 \\
b_{s_{1}} & a_{s_{1}}+a_{f} & b_{f} & 0 \\
0 & b_{f} & a_{f}+a_{s_{2}} & b_{s_{2}} \\
0 & 0 & b_{s_{2}} & a_{s_{2}}-F_{0}
\end{array}\right) .
$$

The transmission $(t)$ and reflection $(r)$ coefficients are given as in Sec. III [Eqs. (11), (12), and (32)]. We get the following expressions for $t$ and $r$, respectively:

$$
t=2 F_{0} \frac{b_{f} b_{s_{1}} b_{s_{2}}}{\Delta^{\prime}}
$$

and

$$
r=\frac{\left[\left(a_{f}+a_{s_{2}}\right)\left(a_{s_{2}}-F_{0}\right)-b_{s_{2}}^{2}\right]\left[\left(a_{f}+a_{s_{1}}\right)\left(a_{s_{1}}+F_{0}\right)-b_{s_{1}}^{2}\right]-b_{f}^{2}\left(a_{s_{2}}-F_{0}\right)\left(a_{s_{1}}+F_{0}\right)}{\Delta^{\prime}},
$$

where $\Delta^{\prime}$ is the determinant of the matrix given in Eq. (32):

$$
\Delta^{\prime}=\left[\left(a_{f}+a_{s_{2}}\right)\left(a_{s_{2}}-F_{0}\right)-b_{s_{2}}^{2}\right]\left[\left(a_{s_{1}}+a_{f}\right)\left(a_{s_{1}}-F_{0}\right)-b_{s_{1}}^{2}\right]-b_{f}^{2}\left(a_{s_{1}}-F_{0}\right)\left(a_{s_{2}}-F_{0}\right) .
$$

In order to get the AIT resonance whatever the solid layer thicknesses, we start from the situation where the structure exhibits a trapped mode (Sec. IV A), namely, $d_{s 1}=d_{s 2}=d_{f}$ and $\theta=\theta_{0}$, then we shift slightly $d_{s 1}$ and $d_{s 2}$ by a small amount $\epsilon$ from $d_{f}$ (for example, $d_{s 1}=d_{f}-\epsilon$ and $d_{s 2}=d_{f}+\epsilon$ ) in order to separate the two transmission zeros induced by the two solid layers. In the next step, we search for the angle $\theta$ that enables to get an antireflection (total transmission). This angle is obtained by vanishing simultaneously the real and imaginary parts of the reflection coefficient [Eq. (34)], namely,

$$
\begin{aligned}
& a_{s_{1}}^{2} a_{s_{2}} \alpha^{\prime}-b_{s_{1}}^{2} \beta^{\prime}-b_{s_{2}}^{2} a_{s_{1}}^{2}+a_{f} a_{s_{1}}\left(a_{s_{2}}^{2}-b_{s_{2}}^{2}\right)-f^{2} a_{f}\left(a_{s_{1}}+a_{s_{2}}\right)-f^{4}=0, \\
& a_{s_{2}} \gamma^{\prime}-a_{s_{1}} \beta^{\prime}+a_{f}\left[\left(a_{s_{1}}^{2}-b_{s_{1}}^{2}\right)-\left(b_{s_{2}}^{2}-b_{s_{2}}^{2}\right)\right]+F^{2}\left(a_{s_{1}}-a_{s_{2}}\right)=0,
\end{aligned}
$$



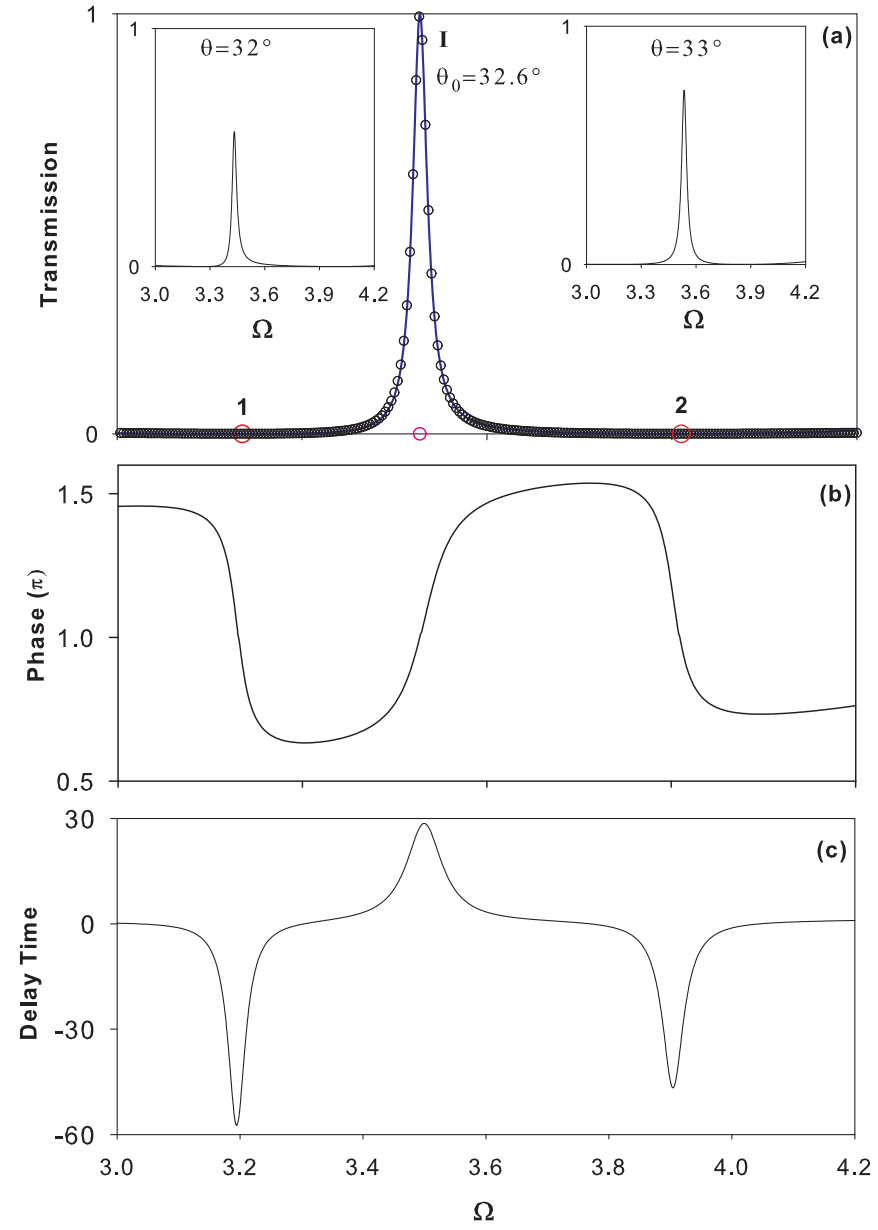

FIG. 11. (a) Comparison between exact results (solid line) and fitted results (open circles) obtained from Eq. (38) of the transmission coefficient versus $\Omega$ for a triple layer characterized by the thicknesses $d_{s_{1}}=1.1 d_{f}$ and $d_{s 2}=0.9 d_{f}$. The incidence angle is fixed to $\theta=$ $32.6^{\circ}$. The insets in (a) give the transmission for $\theta=32^{\circ}$ and $33^{\circ}$. (b), (c) The same as in (a) but for the phase and the delay time, respectively.

where

$$
\begin{aligned}
& \alpha^{\prime}=a_{s_{2}}+a_{f}, \\
& \beta^{\prime}=\left(a_{s_{2}}+a_{f}\right) a_{s_{2}}-b_{s_{2}}^{2}, \\
& \gamma^{\prime}=\left(a_{s_{1}}+a_{f}\right) a_{s_{1}}-b_{s_{1}}^{2} .
\end{aligned}
$$

To illustrate the above analytical results, we have chosen $d_{s 1}=1.1 d_{f}$ and $d_{s 2}=0.9 d_{f}$. The solid and fluid media are Plexiglas and water, respectively. The solutions of Eqs. (36a) and (36b) in the frequency domain $\Omega<4.2$ give $\theta=32.6^{\circ}$ and $37.6^{\circ}$. An example of the transmission amplitude is given in Fig. 11(a) for $\theta_{0}=32.6^{\circ}$ (solid line). One obtains a welldefined EIT resonance around $\Omega=3.5$; this resonance labeled I is squeezed between two transmission zeros indicated by open circles 1 and 2 on the abscissa. Contrary to the behavior of the Fano resonance (Fig. 6), the amplitude of the transmission coefficient falls down rapidly to zero when $\theta$ shifts slightly from $\theta_{0}=32.6^{\circ}$ as it is shown in the inset of Fig. 11(a) for $\theta=32^{\circ}$ and $33^{\circ}$. In order to show that the transparent window is followed by a steep dispersion, we have plotted in Fig. 11(b) the phase of the transmission. As predicted, the two transmission zeros give rise to two phase drops of $\pi$, whereas the phase enhances by $\pi$ around the AIT resonance at $\Omega=3.5$; this behavior is a characteristic of the AIT resonance $[3,4]$. From the phase $\varphi$, one can deduce also the delay time defined as $\tau=\frac{d \varphi}{d \omega}[65,69,70]$. Figure 11(c) clearly shows this negative delay time associated to transmission zeros and a positive delay time associated to the AIT resonance. The negative and positive delay times lead, respectively, to fast and slow sound around the given frequencies where the triple-layer material can be used as supersonic and subsonic materials.

In order to confirm that the resonance in Fig. 11(a) follows the AIT shape, we have sketched in the same figure by open circles the fitted curves obtained from the expression [5]

$$
T=B \frac{\left(\Omega-\Omega_{R}+q_{1} \Gamma\right)^{2}\left(\Omega-\Omega_{R}+q_{1} \Gamma\right)^{2}}{\left(\Omega-\Omega_{R}\right)^{2}+\Gamma^{2}},
$$

where $B=\frac{1}{\left(q_{1} q_{2} \Gamma\right)^{2}} \cdot q_{1}=7.21$ and $q_{2}=-11.94$ are the Fano parameters and $\Gamma=0.052$ (in units of $d_{f} / v_{f}$ ) is the width of the AIT resonance lying at $\Omega_{R}=3.5$. We can notice a very good agreement between the exact results (solid curve) and the fitted results (open circles) obtained from Eq. (38).

An analysis of the displacement field versus the space position (Fig. 12) clearly shows that the modes labeled 1 and 2 in Fig. 11(a) are prohibited from propagation outside the first and the second solid layers, respectively, as it is shown in Figs. 12(a) and 12(b), whereas the AIT mode I [Fig. 12(c)] is well confined inside the triple layer and propagates in the whole structure.

In order to give a better insight about the variation of the transmission rate versus $\Omega$ and $\theta$, we have plotted in Fig. 13 the transmission amplitude (color scale) as well as the dispersion curves of the transmission zeros (white dotted lines) versus $\Omega$ and $\theta$. One can see clearly the existence of only one AIT resonance labeled I squeezed between two transmission zeros labeled 1 and 2 at $\theta=32.6^{\circ}$. Similarly, we found a very sharp AIT resonance labeled II around $\theta=37.6^{\circ}$.

Finally, in Fig. 13, we would like to make a comment when the incidence angle is small. Indeed, one can notice the existence of a broad resonance between the transmission zeros for small values of the incidence angle. Indeed, for $\theta=0^{\circ}$ (normal incidence) the transmission zeros disappear as there is no interaction between transverse and longitudinal waves in the solid layers [62]. In this case, we obtain a large Breight-Wigner (B-W) resonance as it is shown in Fig. 14(a). For $\theta$ close to $0^{\circ}$, Fig. 14(b) shows two abrupt transmission zeros that break the shape of the B-W resonance. However, the latter resonance cannot be qualified either as AIT resonance or as Fano resonance as it cannot be fitted by an equation similar to Fano [Eq. (31)] or AIT formula [Eq. (38)].

In what follows, we shall study the effect of the nature of the solid and fluid layers on the AIT resonance. Indeed, the position, amplitude, and width of the AIT resonance depend on the thickness of the solid layers as it is illustrated in Fig. 15 for $d_{s 2}=0.9 d_{f}$ fixed whereas $d_{s 1}$ is variable. The incidence angle is fixed at $\theta=32.6^{\circ}$. Starting from the thickness $d_{s 1}=1.1 d_{f}$ where the transmission reaches unity [blue curve in Fig. 15(a)], one can notice that as far as $d_{s 1}$ increases the position of the AIT 

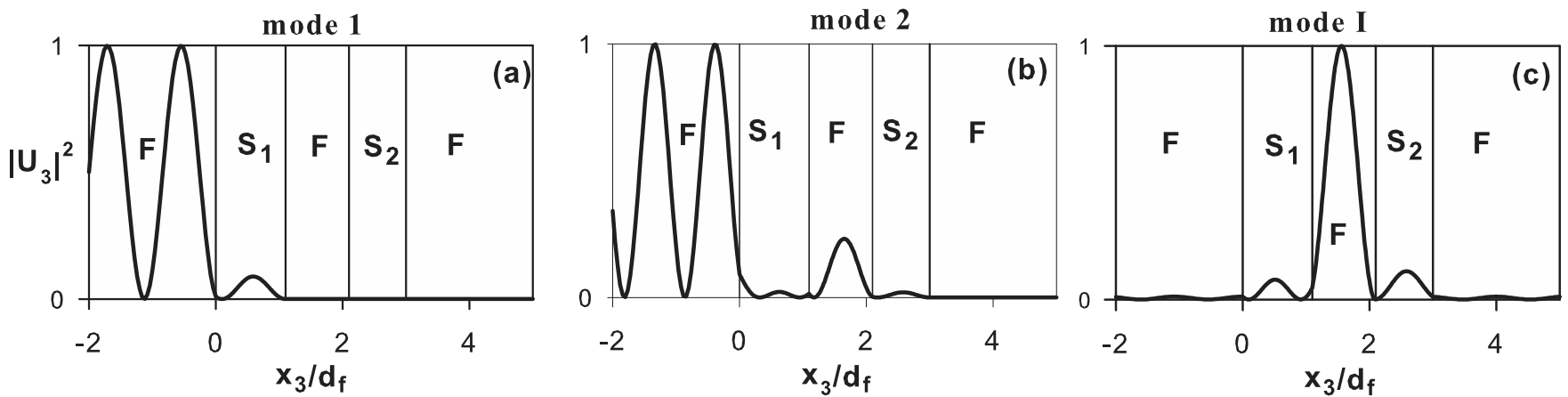

FIG. 12. (a), (b) Square modulus of the displacement field versus the space position for the modes labeled 1, 2 (transmission zeros) in Fig. 11(a). (c) The same as in (a) and (b), but for the mode labeled I (AIT resonance) in Fig. 11(a).

resonance shifts to lower frequencies, its amplitude decreases and its width increases as the separation between the two transmission zeros increases [Fig. 15(a)]. Indeed, the position of the transmission zero induced by the solid layer of thickness $d_{s 2}=0.9 d_{f}$ falls at the frequency indicated by the open circle on the abscissa, whereas the transmission zeros associated to the solid layers of thickness $d_{s 1}=1.1 d_{f}, 1.2 d_{f}$, and $1.3 d_{f}$ shift to lower frequencies as far as $d_{s 1}$ increases. However, by using Eqs. (36a) and (36b), one can find the appropriate angle for each thickness $d_{s 1}$ of the first solid layer that enables to realize a complete transparency. This is illustrated in Fig. 15(b) where the resonances are brought back to unity for the angles indicated in the inset.

The nature of the fluid layer inserted between the solid layers may also affect considerably the AIT resonance. An example is given in Fig. 16(a) where the thicknesses of the layers are the same as in Fig. 11(a). We can see that changing the water layer (solid curve) by the alcohol layer (dashed curve) shifts the AIT resonance to lower frequencies. In addition, the AIT resonance in the case of water layer transforms to a Fano resonance in the case of alcohol layer as the latter falls below

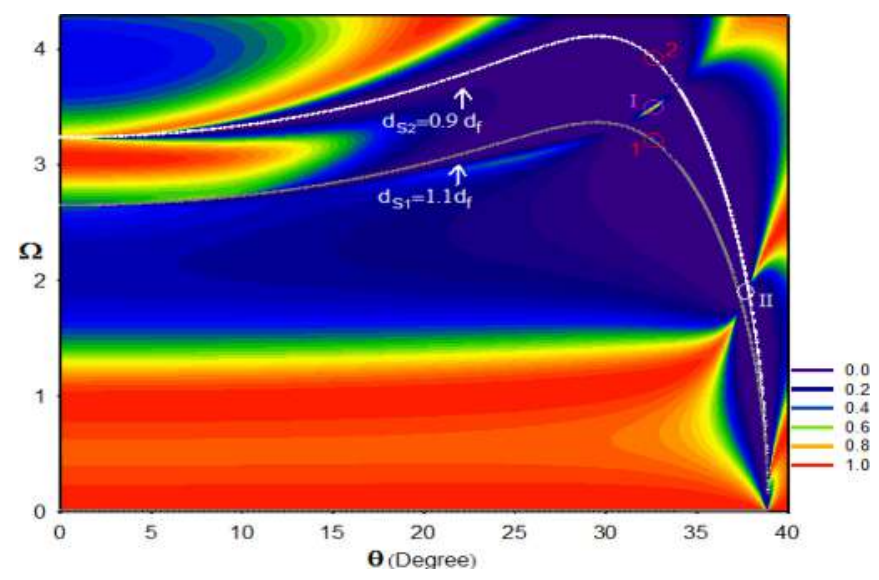

FIG. 13. (a) Variation of the transmission coefficient as a function of $\Omega$ and the angle of incidence $\theta$. Color areas denote different transmission rates. The white dotted curves show the positions of the transmission zeros (total reflection) induced by the first and second solid layers with thicknesses $d_{s_{1}}=1.1 d_{f}$ and $d_{s_{2}}=0.9 d_{f}$, respectively. The solid and fluid media are composed of Plexiglas and water, respectively. the two transmission zeros induced by the solid layers. From Eqs. (36a) and (36b), one can find the appropriate incidence angle $\left(\theta=35.6^{\circ}\right)$ that enables to bring the resonance to unity as it is shown in Fig. 16(b) (dashed curve). In addition, the resonance becomes thinner.

Also, the nature of the solid layers constituting the triple layer may affect the profile of the AIT resonance. An example is given in Fig. 17(a) where we have compared the results of the structure with Plexiglas layers of thicknesses $d_{s_{1}}=1.1 d_{f}$ and $d_{s_{2}}=0.9 d_{f}$ on both sides and the case were the Plexiglas layer of thickness $d_{s_{2}}=0.9 d_{f}$ at the right side is replaced by a PMMA layer. The incidence angle is fixed at $\theta=32.6^{\circ}$. We notice a significant decrease of the amplitude of the AIT resonance from 1 in the case of Plexiglas (solid curve) to 0.25 in the case of PMMA layer (dashed curve) and its shift to higher frequencies. By the help of Eqs. (36a) and (36b), one can determine easily the appropriate incidence angle that enables to bring the AIT resonance to unity in the case of PMMA layer as it is shown in Fig. 17(b) (dashed curve). Let us mention that the effect of dissipation on AIT resonances will be discussed in the Appendix.
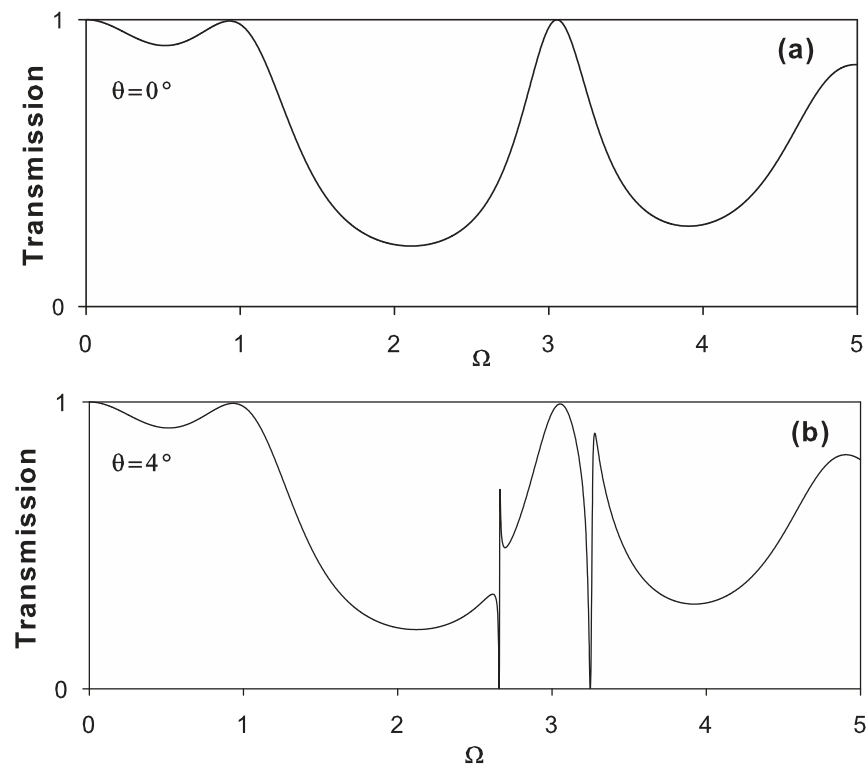

FIG. 14. Transmission coefficient versus $\Omega$ for $\theta=0^{\circ}$ (a) and $\theta=4^{\circ}(\mathrm{b})$. The structure is the same as in Fig. 13 . 

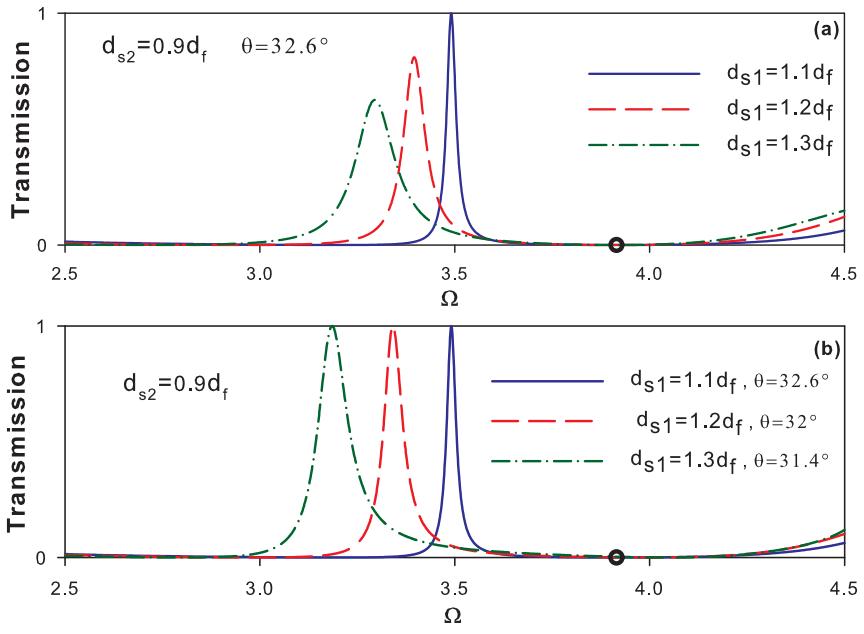

FIG. 15. Variation of the transmission coefficient versus the dimensionless frequency $\Omega$ for $d_{s 2}=0.9 d_{f}$ fixed whereas $d_{s 1}$ is variable: $d_{s 1}=1.1 d_{f}$ (full curve), $d_{s 1}=1.2 d_{f}$ (dashed curve), and $d_{s 1}=1.3 d_{f}$ (dashed-dotted curve). The incidence angle is fixed at $\theta=32.6^{\circ}$. (b) The same as in (a) but the AIT resonances are brought back to unity by choosing appropriately the angles of incidence as indicated in the inset. The open circle on the abscissa indicates the position of the transmission zero induced by the solid layer of thickness $d_{s 2}=0.9 d_{f}$.

\section{SOLID-FLUID MULTILAYERED STRUCTURE}

The results presented above on Fano and AIT resonances may have interesting consequences for understanding the band-gap structures and the transmission properties in periodic solid-fluid layers. We shall consider a 1D phononic crystal composed of alternating solid and fluid layers and we shall
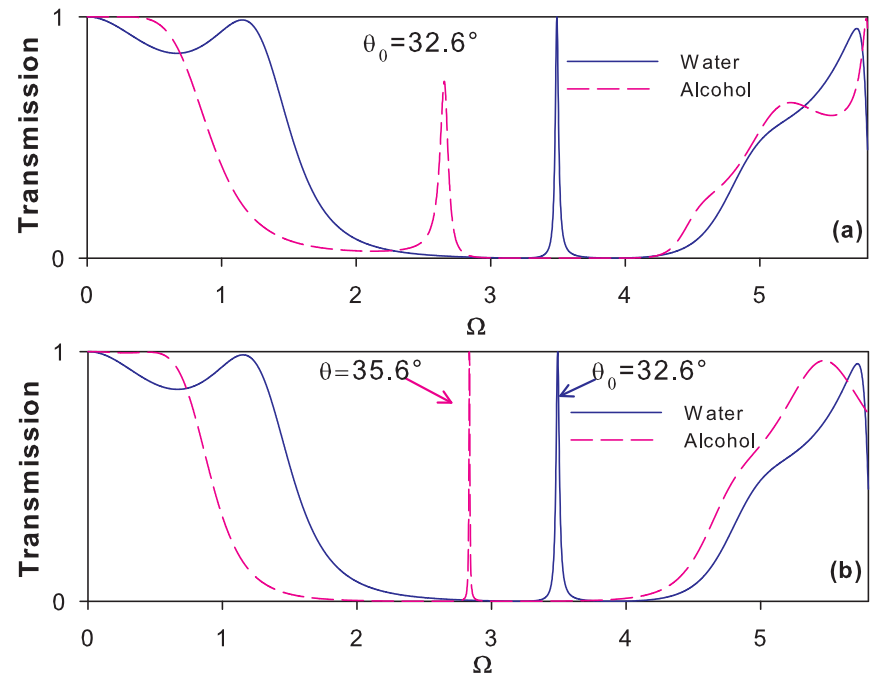

FIG. 16. (a) Variation of the transmission coefficient versus the dimensionless frequency $\Omega$ for the same structure as in Fig. 11 (full curve) and when the water layer is replaced by an alcohol layer (dashed curve). (b) The same as in (a) but the Fano resonance in (a) is brought back to a unitary AIT resonance by choosing appropriately the incidence angle $\theta=35.6^{\circ}$.
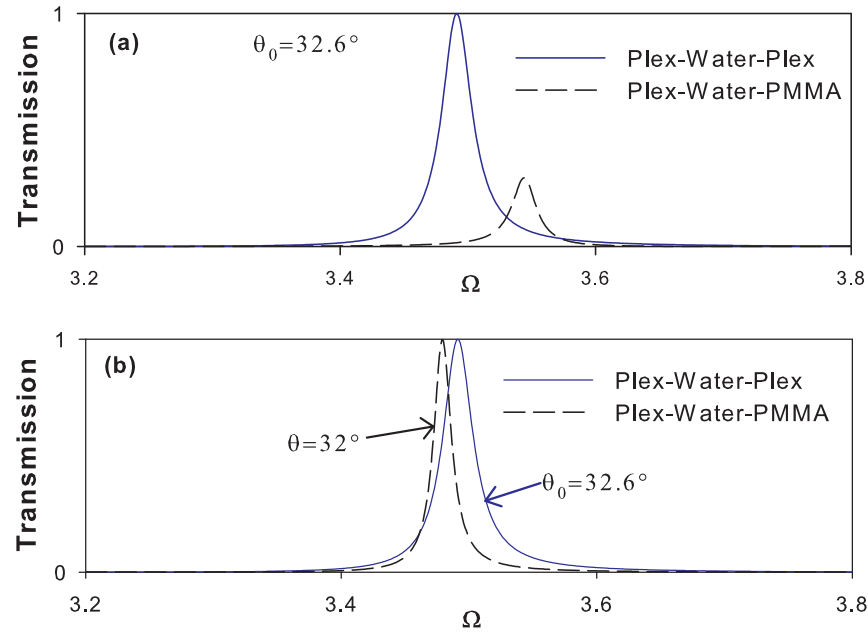

FIG. 17. Same as in Fig. 16, but one Plexiglas layer is replaced by a PMMA layer.

demonstrate how the trapped modes and Fano resonances may give rise to flat and nearly flat bands in the band structures.

Let us first recall the expressions of the dispersion relation and the transmission coefficient for a solid-fluid phononic crystal. Consider an infinite superlattice (SL) made of a periodic repetition of solid-fluid layers. The Green's function is obtained by a linear juxtaposition of the $2 \times 2$ matrices [Eqs. (4) and (6)] at the different interfaces. A tridiagonal matrix can thus be obtained. By using the Bloch theorem and Eq. (2), we obtain the following expression for the dispersion relation of an infinite SL [52]:

$$
\cos (K D)=-\frac{\left(a_{s}^{2}-b_{s}^{2}+a_{f}^{2}-b_{f}^{2}+2 a_{s} a_{f}\right)}{2 b_{s} b_{f}},
$$

where $K$ is the Bloch wave vector and $D=d_{s}+d_{f}$ is the period of the SL. On the other hand, the transmission coefficient along a finite structure composed of $N$ solid-fluid bilayers can be written as follows [52]:

$$
t_{N}=2 F \frac{b(N)}{a^{2}(N)-b^{2}(N)+F^{2}-2 F a(N)},
$$

where

$$
\begin{aligned}
& a(N)=\left(\frac{Y_{1}}{a_{s}+a_{f}}\right)\left[1-b_{s} b_{f}\left(t-\frac{1}{t}\right) \frac{Y_{1}}{\Lambda}\right], \\
& b(N)=b_{s} b_{f}\left(t-\frac{1}{t}\right) \frac{Y_{1} Y_{2}}{a_{s}+a_{f}} t^{N-1},
\end{aligned}
$$

with $\Lambda=Y_{1}^{2}-Y_{2}^{2} t^{2(N-1)}, \quad Y_{1}=b_{f}^{2}-a_{f}^{2}-a_{f} a_{s}+b_{s} b_{f} t$,

$$
Y_{2}=a_{f} b_{s}-a_{s} b_{f} t \text { and } t=e^{j K D} .
$$

Figure 18 gives the dispersion curves (hatched areas) for an infinite SL made of Plexiglas and water layers. In this figure, we plotted the dimensionless frequency $\Omega=\omega d_{f} / v_{f}$ as a function of the incidence angle $\theta$. The thicknesses of solid and fluid layers are assumed to be equal $d_{s}=d_{f}$. The dotted lines inside the allowed bands represent the dispersion curves obtained from the peaks of transmission (i.e., zero reflection) for a finite SL composed of $N=6$ solid layers. The blue circles 


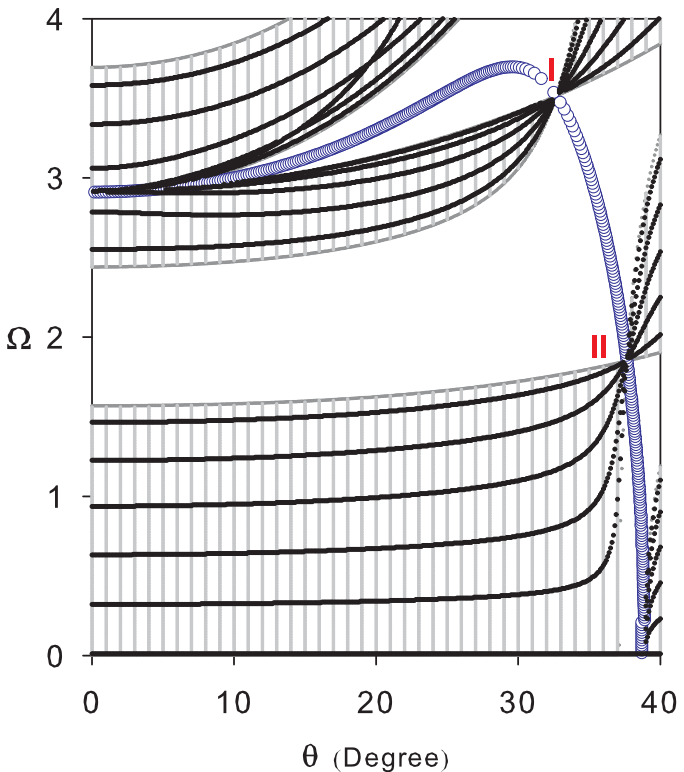

FIG. 18. Dispersion curves $\Omega$ versus the incidence angle $\theta$ for an infinite superlattice (SL) made of Plexiglas and water layers (hatched areas). The thin solid curves show the dispersion curves obtained from the maxima of the transmission (zero reflection), whereas the open circles give the position of the total reflection (transmission zero).

give the positions of the transmission zeros (total reflection). One can notice a shrinking of the discrete branches and pass bands when they intercept the transmission zero branch around $\left(\theta=32.6^{\circ}, \Omega=3.5\right)$ and $\left(\theta=37.2^{\circ}, \Omega=1.85\right)$ labeled I and II, respectively. This property of the shrinking of the modes is a characteristic of solid-fluid SLs and is without analog in their counterpart solid-solid SLs [52].

Indeed, at the crossing points I and II, there is a degeneracy of the modes ( $N=5$ times in this case) giving rise to dispersionless flat bands with zero group velocity $\left(v_{g}=0\right)$ around $\Omega=3.5$ and 1.85 for $\theta=32.6^{\circ}$ and $37.2^{\circ}$, respectively. Figures 19(a) and 19(b) give the variation of the transmission coefficient versus $\Omega$ for a finite SL composed of $N=2$ [Fig. 19(a)] and $N=4$ [Fig. 19(b)]. The position of the BIC mode (Fig. 6) is indicated by a filled circle on the abscissa of Figs. 19(a) and 19(b). One can notice that when $N$ increases, the width of the gap around $\Omega=3.5$ increases [Fig. 19(b)]. The transmission gap coincides with the band-gap structure of the infinite SL [Fig. 19(c)] where the BIC mode leads to a zero bandwidth around $\Omega=3.5$ in the first Brillouin zone. For $\theta>$ $\theta_{0}$ [Figs. 19(d)-19(f)], the Fano resonance obtained for $N=2$ [Fig. 19(d)] transforms to three modes within a narrow band for $N=4$ [Fig. 19(e)] and a nearly flat band with positive group velocity $\left(v_{g}>0\right)$ in the case of an infinite SL [Fig. 19(f)]. If $\theta<\theta_{0}$, Figs. 19(g), 19(h), and 19(k) give the same behaviors as in Figs. 19(d), 19(e), and 19(f) but with a nearly flat band with negative group velocity $\left(v_{g}<0\right)$ [Fig. 19(k)]. These results clearly show that one can change the shape of the dispersion curves by detuning the incidence angle around $\theta_{0}=32.6^{\circ}$. Therefore, solid-fluid multilayers can be designed to exhibit forward or backward slow-sound propagation. Let us mention that the existence of zero-bandwidth property has been also studied in photonic crystals [71].
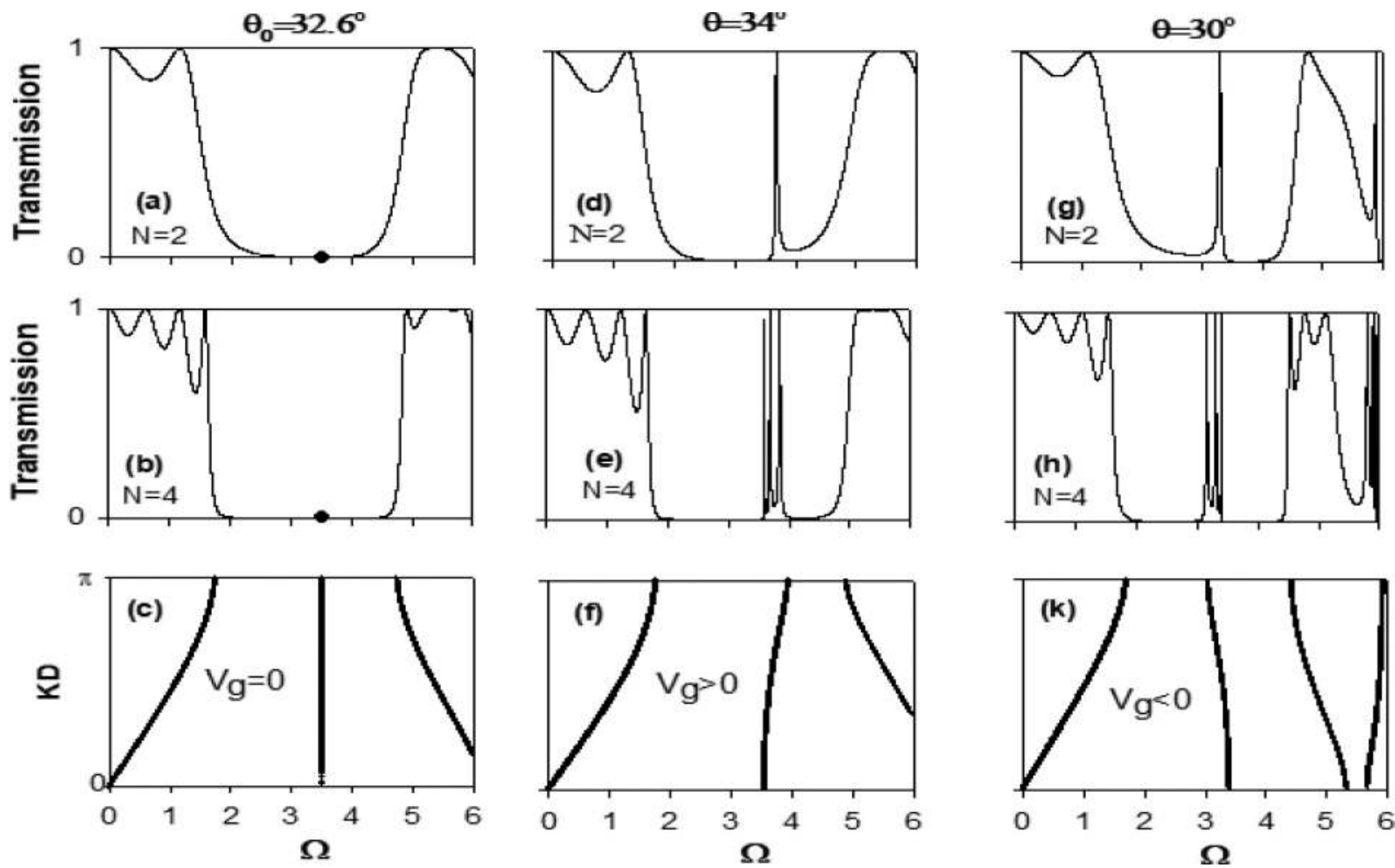

FIG. 19. Variation of the transmission coefficients as a function of $\Omega$ for a finite SL composed of $N=2[(\mathrm{a}),(\mathrm{d})$, and (g)] and $N=4[(\mathrm{~b})$, (e), and (h)] periods. The left, middle, and right panels correspond to incident angles: $\theta=32.6^{\circ}, 34^{\circ}$, and $30^{\circ}$, respectively. (c), (f), (k) Give the dispersion curves (i.e., $\Omega$ versus the dimensionless Bloch wave vector $K D$ ) inside the first Brillouin zone (i.e., $0<K D<\pi$ ). 


\section{CONCLUSION}

We have investigated theoretically and numerically the possibility of existence of the acoustical analog of Fano and EIT resonances in simple structures made of a single solid layer or a solid-fluid-solid triple layer inserted between two fluids. This study has been performed by means of the Green's function method which enables to determine analytically the transmission and reflection coefficients as well as the dispersion relations, density of states, and displacement fields. We have demonstrated analytically that these structures may exhibit a bound state in continuum for a given incidence angle as a consequence of the combination of the transmission zeros induced by the solid layers and the eigenmodes of a symmetric solid layer or a triple solid-fluid-solid layer with free boundary conditions. This trapped mode is independent of the surrounding fluid media and behaves as a standing mode of the system without leakage. By adjusting the incidence angle and the width of the solid layers, one can create a sharp asymmetric resonance (transparency window) at the vicinity of a transmission zero, the so-called Fano resonance or a sharp resonance squeezed between two transmission zeros, the so-called AIT resonance. These two resonances have been written following a Fano-type form which enables to deduce the Fano parameter that describes their asymmetry. The dependence of these resonances on the nature of the solid and fluid layers is also discussed. In addition to the transmission amplitude, we have also studied the phase of the transmission which enabled us to determine the delay time. In particular, we have shown that transmission zeros give rise to phase drops of $\pi$ and therefore negative delay times leading to supersonic phenomena. Whereas, the resonances give rise to an enhancement of the delay time and therefore subsonic phenomena. Finally, we have shown that trapped modes give rise to flat bands with zero group velocity in a solid-fluid phononic crystal and Fano resonances give rise to nearly flat bands with positive or negative group velocities. These structures may have interesting applications in designing acoustic mirrors and acoustic filters as well as supersonic and subsonic materials.

In all the above results, we have considered lossless materials. However, the effect of absorption can affect considerably the shape of Fano and AIT resonances. This point has been addressed in the Appendix where the intensity and width of the resonances can change significantly for lossy media. However, the shape of these resonances still remains visible for low-absorbing media. The shapes of the resonances (see Appendix) are similar to those we have obtained recently in photonic crystals based on lossy coaxial cables $[36,37]$.

\section{APPENDIX: EFFECT OF ABSORPTION}

In this appendix, we shall consider the effect of absorption on Fano and AIT resonances discussed in Secs. III and IV. The dissipation can be included by adding a small imaginary part to the transverse and longitudinal velocities of sound in solid layers such that $C_{t}^{\prime}=C_{t}(1+\varepsilon)$ and $C_{l}^{\prime}=C_{l}(1+\varepsilon)$ $[55,56]$. The dissipation induced by water layers is negligible
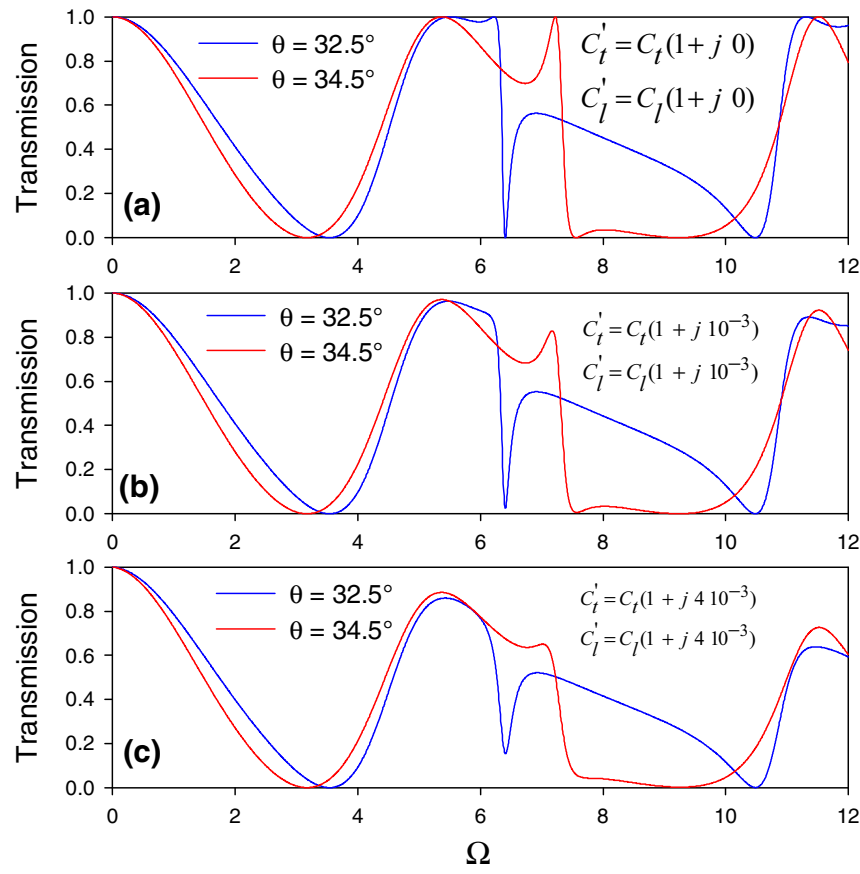

FIG. 20. Variation of the transmission coefficients as a function of $\Omega=\frac{\omega d_{s}}{v_{0}}$ for the structure depicted in Fig. 1 for $\theta=32.5^{\circ}$ (blue curve) and $\theta \stackrel{v_{0}}{=} 34.5^{\circ}$ (red curve). The curves are plotted for a Plexiglas layer without absorption (a), small absorption (b), and high absorption (c).

if we assume to work in the $\mathrm{MHz}$ range with millimeter-size layers [55,56]. Figure 20 gives the transmission coefficients
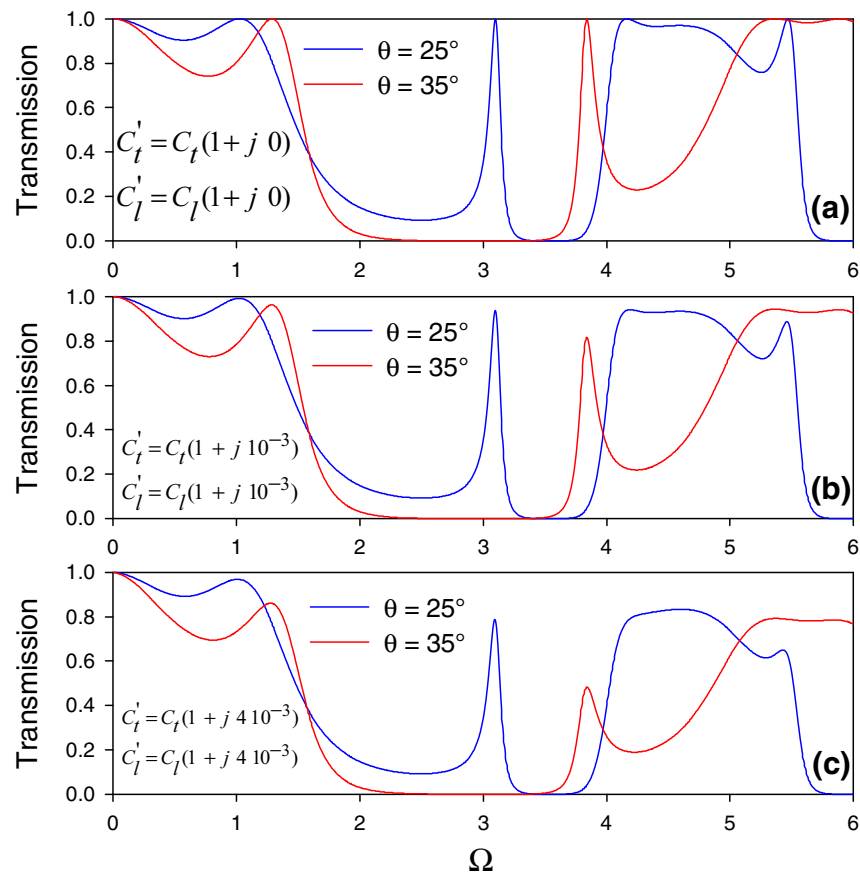

FIG. 21. Variation of the transmission coefficients as a function of $\Omega=\frac{\omega d_{f}}{v_{f}}$ for a solid-fluid-solid triple layer (Fig. 5) where the thicknesses of the layers are chosen such that $d_{s_{1}}=d_{s 2}=d_{f}$. The incidence angle is taken as $\theta=25^{\circ}$ (blue curve) and $\theta=35^{\circ}$ (red curve). The curves are plotted for a Plexiglas layer without absorption (a), small absorption (b), and high absorption (c). 


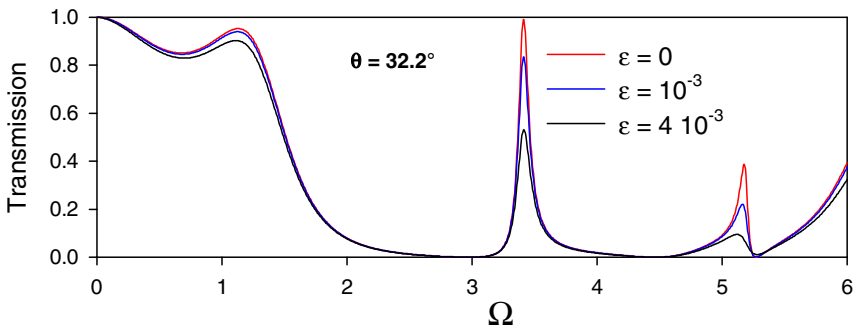

FIG. 22. Variation of the transmission coefficients as a function of $\Omega=\frac{\omega d_{f}}{v_{f}}$ for a solid-fluid-solid triple layer (Fig. 5) where the thicknesses of the layers are chosen such that $d_{s_{1}}=1.2 d_{f}$ and $d_{s 2}=$ $0.8 d_{f}$. The incidence angle is taken as $\theta=32.2^{\circ}$. The curves are plotted for a Plexiglas layer without absorption (red curve), small absorption (blue curve), and high absorption (black curve).

as a function of $\Omega=\frac{\omega d_{s}}{v_{s}}$ for a single solid layer inserted in a fluid (Fig. 1) for $\theta=32.5^{\circ}$ (blue curve) and $\theta=34.5^{\circ}$ (red curve). The curves are similar to those in Figs. 3(a) and 3(c), but the incidence angles are chosen slightly far from the BIC mode in order to broaden the Fano resonances. In Fig. 20, the Plexiglas layer is considered without absorption [i.e., $\varepsilon=0$, Fig. 20(a)], small absorption [i.e., $\varepsilon=10^{-3}$, Fig. 20(b)], and high absorption [i.e., $\varepsilon=410^{-3}$, Fig. 20(c)]. One can notice that as far as the absorption increases, the intensity of Fano resonances around $\Omega=7$ decreases and their width increases.
In particular, the shape of Fano resonances undergoes alteration for high dissipation [Fig. 20(c)].

Figure 21 gives the transmission coefficients as a function of $\Omega=\frac{\omega d_{f}}{v_{f}}$ for a solid-fluid-solid triple layer inserted in a fluid (Fig. 5) for $\theta=25^{\circ}$ (blue curve) and $\theta=35^{\circ}$ (red curve). The thicknesses of the layers are chosen such that $d_{s_{1}}=d_{s 2}=d_{f}$. The curves are similar to those in Figs. 6(c) and 6(e), but the incidence angles are chosen slightly far from the BIC mode in order to broaden the Fano resonances. In Fig. 21, the Plexiglas layer is considered without absorption [i.e., $\varepsilon=0$, Fig. 21(a)], small absorption [i.e., $\varepsilon=10^{-3}$, Fig. 21(b)], and high absorption [i.e., $\varepsilon=410^{-3}$, Fig. 21(c)]. Even though the intensity of Fano resonances decreases when the dissipation increases, their shape remains of Fano type even for high dissipation [Fig. 21(c)].

Figure 22 shows the effect of absorption on AIT resonance. The triple layer is similar to the one studied in Fig. 9, but the thicknesses of the layers are chosen such that $d_{s_{1}}=1.2 d_{f}$ and $d_{s 2}=0.8 d_{f}$ in order to enlarge the AIT resonance. The incidence angle is fixed to $\theta=32.2^{\circ}$. The curves are plotted for a Plexiglas layer without absorption (red curve), small absorption (blue curve), and high absorption (black curve). Here, also, we remark that even though the intensity of the AIT resonance decreases when the dissipation increases, its shape remains of AIT type even for high dissipation [black curve in Fig. 22(c)]. It is worth mentioning that experimental value of dissipation $\varepsilon$ in the case of Plexiglas layers is around $410^{-3}$ (see Ref. [55]).
[1] G. Breit and E. Wigner, Phys. Rev. 49, 519 (1936).

[2] U. Fano, Phys. Rev. 124, 1866 (1961).

[3] A. E. Miroshnichenko, S. Flach, and Y. S. Kivshar, Rev. Mod. Phys. 82, 2257 (2010).

[4] S. E. Harris, Phys. Today 50(7), 36 (1997).

[5] M. Fleischhauer, A. Imamoglu, and J. P. Marangos, Rev. Mod. Phys. 77, 633 (2005).

[6] Y. Plotnik, O. Peleg, F. Dreisow, M. Heinrich, S. Nolte, A. Szameit, and M. Segev, Phys. Rev. Lett. 107, 183901 (2011).

[7] E. N. Bulgakov and A. F. Sadreev, Phys. Rev. B 78, 075105 (2008).

[8] M. I. Molina, A. E. Miroshnichenko, and Y. S. Kivshar, Phys. Rev. Lett. 108, 070401 (2012).

[9] D. C. Marinica, A. G. Borisov, and S. V. Shabanov, Phys. Rev. Lett. 100, 183902 (2008).

[10] S. Weimann, Y. Xu, R. Keil, A. E. Miroshnichenko, A. Tünnermann, S. Nolte, A. A. Sukhorukov, A. Szameit, and Y. S. Kivshar, Phys. Rev. Lett. 111, 240403 (2013).

[11] G. Corrielli, G. Della Valle, A. Crespi, R. Osellame, and S. Longhi, Phys. Rev. Lett. 111, 220403 (2013).

[12] J. Mur-Petit and R. A. Molina, Phys. Rev. B 90, 035434 (2014).

[13] R. A. Vicencio, C. Cantillano, L. Morales-Inostroza, B. Real, C. Mejía-Cortés, S. Weimann, A. Szameit, and M. I. Molina, Phys. Rev. Lett. 114, 245503 (2015).

[14] C. W. Hsu, Bo Zhen, J. Lee, S.-L. Chua, S. G. Johnson, J. D. Joannopoulos, and M. Soljačić, Nature (London) 499, 188 (2013).

[15] L.-S. Li and H.-C. Yin, Sci. Rep. 6, 26988 (2016).
[16] J. von Neumann and E. Wigner, Phys. Z. 30, 465 (1929).

[17] C. W. Hsu, Bo Zhen, A. D. Stone, J. D. Joannopoulos, and M. Soljačić, Nat. Rev. Mater. 1, 16048 (2016).

[18] V. A. Fedotov, M. Rose, S. L. Prosvirnin, N. Papasimakis, and N. I. Zheludev, Phys. Rev. Lett. 99, 147401 (2007).

[19] S. Zhang, D. A. Genov, Y. Wang, M. Liu, and X. Zhang, Phys. Rev. Lett. 101, 047401 (2008).

[20] N. Liu, L. Langguth, T. Weiss, J. Kästel, M. Fleischhauer, T. Pfau, and H. Giessen, Nat. Mater. 8, 758 (2009).

[21] B. Lukyanchuk, N. I. Zheludev, S. A. Maier, N. J. Halas, P. Nordlander, H. Giessen, and C. T. Chong, Nat. Mater. 9, 707 (2010).

[22] P. Tassin, L. Zhang, T. Koschny, E. N. Economou, and C. M. Soukoulis, Phys. Rev. Lett. 102, 053901 (2009).

[23] R. Taubert, M. Hentshel, J. Kastel, and H. Giessen, Nano Lett. 12, 1367 (2012).

[24] J. Gu, R. Singh, X. Liu, X. Zhang, Y. Ma, S. Zhang, S. A. Maier, Z. Tian, A. K. Azad, H.-T. Chen et al., Nat. Commun. 3, 1151 (2012).

[25] S. Fan and J. D. Joannopoulos, Phys. Rev. B 65, 235112 (2002).

[26] M. F. Yanik, W. Suh, Z. Wang, and S. Fan, Phys. Rev. Lett. 93, 233903 (2004).

[27] X. Yang, M. Yu, D.-L. Kwong, and C. W. Wong, Phys. Rev. Lett. 102, 173902 (2009).

[28] Y. Huo, S. Sandhu, J. Pan, N. Stuhrmann, M. L. Povinelli, J. M. Kahn, J. S. Harris, M. M. Fejer, and S. Fan, Opt. Lett. 36, 1482 (2011). 
[29] Y. Sato, Y. Tanaka, J. Upham, Y. Takahashi, T. Asano, and S. Noda, Nat. Photonics 6, 56 (2012).

[30] L. Maleki, A. B. Matsko, A. A. Savchenkov, and V. S. Ilchenko, Opt. Lett. 29, 626 (2004).

[31] D. D. Smith, H. Chang, K. A. Fuller, A. T. Rosenberger, and R. W. Boyd, Phys. Rev. A 69, 063804 (2004).

[32] K. Totsuka, N. Kobayashi, and M. Tomita, Phys. Rev. Lett. 98, 213904 (2007).

[33] C. H. Raymond Ooi and C. H. Kam, Phys. Rev. B 81, 195119 (2010).

[34] B.-B. Li, Y.-F. Xiao, C.-L. Zou, X.-F. Jiang, Y.-C. Liu, F.-W. Sun, Y. Li, and Q. Gong, Appl. Phys. Lett. 100, 021108 (2012).

[35] W. Ding, B. Lukyanchuk, and C.-W. Qiu, Phys. Rev. A 85, 025806 (2012).

[36] A. Mouadili, E. H. El Boudouti, A. Soltani, A. Talbi, A. Akjouj, and B. Djafari-Rouhani, J. Appl. Phys. 113, 164101 (2013).

[37] A. Mouadili, E. H. El Boudouti, A. Soltani, A. Talbi, B. DjafariRouhani, A. Akjouj, and K. Haddadi, J. Phys.: Condens. Matter 26, 505901 (2014).

[38] E. H. El Boudouti, T. Mrabti, H. Al-Wahsh, B. Djafari-Rouhani, A. Akjouj, and L. Dobrzynski, J. Phys.: Condens. Matter 20, 255212 (2008).

[39] A. Santillan and S. I. Bozhevolnyi, Phys. Rev. B 84, 064304 (2011).

[40] W. Tan, C. Z. Yang, H. S. Liu, Z. G. Wang, H. Q. Lin, and H. Chen, Europhys. Lett. 97, 24003 (2012).

[41] G. Yu and X. J. Wang, J. Appl. Phys. 115, 044913 (2014).

[42] A. Merkel, G. Theocharis, O. Richoux, V. Romero-Garcìa, and V. Pagneux, Appl. Phys. Lett. 107, 244102 (2015).

[43] H. Stefan, W. Koch, and L. Nannen, J. Fluid Mech. 692, 257 (2012).

[44] F. Liu, M. Ke, A. Zhang, W. Wen, J. Shi, Z. Liu, and P. Sheng, Phys. Rev. E 82, 026601 (2010).

[45] M. Amin, A. Elayouch, M. Farhat, M. Addouche, A. Khelif, and H. Bac, J. Appl. Phys. 118, 164901 (2015).

[46] C. Goffaux, J. Sánchez-Dehesa, A. LevyYeyati, P. Lambin, A. Khelif, J. O. Vasseur, and B. Djafari-Rouhani, Phys. Rev. Lett. 88, 225502 (2002).

[47] D. Nardi, M. Travagliati, M. E. Siemens, Q. Li, M. M. Murnane, H. C. Kapteyn, G. Ferrini, F. Parmigiani, and F. Banfi, Nano Lett. 11, 4126 (2011).

[48] Y. Jin, E. H. EI Boudouti, Y. Pennec, and B. Djafari-Rouhani, J. Phys. D: Appl. Phys. 50, 425304 (2017).
[49] Y.-X. Xiao, G. Ma, Z.-Q. Zhang, and C. T. Chan, Phys. Rev. Lett. 118, 166803 (2017).

[50] A. A. Lyapina, D. N. Maksimov, A. S. Pilipchuk, and A. F. Sadreev, J. Fluid Mech. 780, 370 (2015).

[51] L. Dobrzynski, E. H. El Boudouti, A. Akjouj, Y. Pennec, H. Al-Wahsh, G. Lévêque, and B. Djafari-Rouhani, Phononics (Elsevier, Amsterdam, 2017).

[52] Y. El Hassouani, E. H. El Boudouti, B. Djafari-Rouhani, and H. Aynaou, Phys. Rev. B 78, 174306 (2008).

[53] H. Sanchis-Alepuz, Y. A. Kosevich, and J. Sánchez-Dehesa, Phys. Rev. Lett. 98, 134301 (2007).

[54] Z. Sai, Z. Yu, and G. Xiao-Wei, Chin. Phys. B 23, 124301 (2014).

[55] R. James, S. M. Woodley, C. M. Dyer, and F. Humphrey, J. Acoust. Soc. Am. 97, 2041 (1995).

[56] M. Shen and W. Cao, Appl. Phys. Lett. 75, 3713 (1999).

[57] B. Liang, X. S. Guo, J. Tu, D. Zhang, and J. C. Cheng, Nat. Mater. 9, 989 (2010).

[58] S. Zhang, Y. Zhang, Y. Guo, Y. Leng, W. Feng, and W. Cao, Phys. Rev. Appl. 5, 034006 (2016).

[59] S. Villa-Arango, R. Torres, P. A. Kyriacou, and R. Lucklum, Measurement 102, 20 (2017).

[60] E. H. El Boudouti, B. Djafari-Rouhani, A. Akjouj, and L. Dobrzynski, Surf. Sci. Rep. 64, 471 (2009).

[61] S. Mizuno, Jpn. J. Appl. Phys. 55, 017302 (2016).

[62] L. M. Brekhovskikh, Waves in Layered Media (Academic, New York, 1981).

[63] I. A. Veres, T. Berer, C. Grünsteidl, and P. Burgholzer, Ultrasonics 54, 759 (2014).

[64] B. Djafari-Rouhani and L. Dobrzynski, J. Phys.: Condens. Matter 5, 8177 (1993)

[65] M. L. H. Lahlaouti, A. Akjouj, B. Djafari-Rouhani, L. Dobrzynski, M. Hammouchi, E. H. El Boudouti, A. Nougaoui, and B. Kharbouch, Phys. Rev. B 63, 035312 (2001).

[66] M. L. Ladrón de Guevara, F. Claro, and P. A. Orellana, Phys. Rev. B 67, 195335 (2003).

[67] Khee-Kyun Voo and Chon Saar Chu, Phys. Rev. B 72, 165307 (2005).

[68] S. P. Shipman and S. Venakides, Phys. Rev. E 71, 026611 (2005).

[69] M. Buttiker and R. Landauer, Phys. Rev. Lett. 49, 1739 (1982).

[70] E. H. Hauge and J. A. Stovneng, Rev. Mod. Phys. 61, 917 (1989).

[71] F. J. Rodriguez-Fortuno, B. Tomas-Navarro, C. Garcia-Meca, R. Ortuno, J. Marti, and A. Martinez, Phys. Rev. B 81, 233101 (2010). 